The University of San Francisco

USF Scholarship: a digital repository @ Gleeson Library |

Geschke Center

2011

\title{
Impacts of Dark Stars on Reionization and Signatures in the Cosmic Microwave Background
}

P Scott

E Roebber

G Holder

Aparna Venkatesan

University of San Francisco, avenkatesan@usfca.edu

P Gondolo

See next page for additional authors

Follow this and additional works at: http://repository.usfca.edu/phys

Part of the Astrophysics and Astronomy Commons, and the Physics Commons

\section{Recommended Citation}

Scott P, Roebber E, Holder G, Venkatesan A, Gondolo P, Pierpaoli E. Impacts of dark stars on reionization and signatures in the cosmic microwave background. Astrophysical Journal. December 1, 2011;742(2).

This Article is brought to you for free and open access by the College of Arts and Sciences at USF Scholarship: a digital repository @ Gleeson Library | Geschke Center. It has been accepted for inclusion in Physics and Astronomy by an authorized administrator of USF Scholarship: a digital repository @

Gleeson Library | Geschke Center. For more information, please contact repository@usfca.edu. 
Authors

P Scott, E Roebber, G Holder, Aparna Venkatesan, P Gondolo, and E Pierpaoli 


\title{
IMPACTS OF DARK STARS ON REIONIZATION AND SIGNATURES IN THE COSMIC MICROWAVE BACKGROUND
}

\author{
Pat Scott $^{1}$, Aparna Venkatesan ${ }^{2}$, Elinore Roebber $^{1}$, Paolo Gondolo $^{3}$, Elena Pierpaoli $^{4}$, And Gil Holder $^{1}$ \\ ${ }^{1}$ Department of Physics, McGill University, Montreal, QC H3A 2T8, Canada; patscott@physics.megill.ca \\ ${ }^{2}$ Department of Physics and Astronomy, University of San Francisco, San Francisco, CA 94117, USA; avenkatesan@usfca.edu \\ ${ }^{3}$ Department of Physics and Astronomy, University of Utah, Salt Lake City, UT 84112, USA \\ ${ }^{4}$ Department of Physics and Astronomy, University of Southern California, Los Angeles, CA 90089, USA \\ Received 2011 July 8; accepted 2011 August 22; published 2011 November 15
}

\begin{abstract}
We perform a detailed and systematic investigation of the possible impacts of dark stars on the reionization history of the universe, and its signatures in the cosmic microwave background (CMB). We compute hydrogen reionization histories, CMB optical depths, and anisotropy power spectra for a range of stellar populations including dark stars. If dark stars capture large amounts of dark matter (DM) via nuclear scattering, reionization can be substantially delayed, leading to decreases in the integrated optical depth to last scattering and large-scale power in the EE polarization power spectrum. Using the integrated optical depth observed by the Wilkinson Microwave Anistropy Probe seven-year mission, in our canonical reionization model we rule out the section of parameter space where dark stars with high scattering-induced capture rates tie up $\gtrsim 90 \%$ of all the first star-forming baryons, and live for $\gtrsim 250$ Myr. When nuclear scattering delivers only moderate amounts of DM, reionization can instead be sped up slightly, modestly increasing the CMB optical depth. If dark stars do not obtain any DM via nuclear scattering, effects on reionization and the CMB are negligible. The effects of dark stars on reionization and its CMB markers can be largely mimicked or compensated for by changes in the existing parameters of reionization models, making dark stars difficult to disentangle from astrophysical uncertainties, but also widening the range of standard parameters in reionization models that can be made consistent with observations.
\end{abstract}

Key words: cosmic background radiation - dark ages, reionization, first stars - dark matter - stars: Population III

Online-only material: color figures

\section{INTRODUCTION}

At a redshift of $z \sim 1100$, the bulk of electrons and protons in the universe recombined to form neutral hydrogen. This cleared the way for thermal photons to free-stream away from the resulting surface of last scattering, forming what we now see as the cosmic microwave background (CMB; see, e.g., Hu \& Dodelson 2002; Samtleben et al. 2007). A prolonged period of darkness ensued, until the first sources of hard ionizing radiation forming within galaxies appeared at redshifts $z \lesssim 30$ (Gnedin 2000; Ciardi et al. 2000; Bromm et al. 2001; Wyithe \& Loeb 2003; Schaerer 2002; Venkatesan et al. 2003; Benson et al. 2006; Loeb 2009). These sources are thought to have reionized the neutral gas in the intergalactic medium (IGM). This process was completed by $z \sim 7$ (Fan et al. 2006; Dawson et al. 2007), with the IGM subsequently remaining in the fully ionized state we see it in today.

The process of reionization is expected to have left its imprint on a number of cosmological observables. The CMB is sensitive to the total optical depth to the surface of last scattering; any free electrons between us and the surface will have scattered CMB photons before they could reach us, modifying the anisotropy power spectra we observe today. Similarly, the changing distribution of neutral hydrogen during reionization can be mapped using its ground-state hyperfine transition (Furlanetto et al. 2006; Chen \& Miralda-Escudé 2008), corresponding to a rest-frame wavelength of $21 \mathrm{~cm}$. The signal is expected to be weak because this is a forbidden line, so the transition probability is small. A multitude of upcoming experiments hope to detect it nonetheless (Morales \& Wyithe 2010); these include the Low-Frequency (LOFAR; Harker et al.
2010), Murchison Wide-Field (MWA; Mitchell et al. 2010), and Square Kilometer Arrays (SKA; Carilli 2008).

The first ionizing sources are generally thought to be the first stars (see, e.g., Gnedin 2000; Bromm et al. 2001; Venkatesan et al. 2003; Tumlinson et al. 2004; Wise \& Abel 2008), referred to as Population (Pop) III. The terms Pop III and Pop II are typically interpreted as broad distinctions based on compositionrespectively, they represent stars that are metal-free and metalpoor. Pop III stars consist entirely of primordial hydrogen and helium synthesized in the big bang, and so are likely to have a mass function weighted toward higher stellar masses than that of Pop II, due to the absence of metal lines, which allow efficient gas cooling and cloud fragmentation in metalenriched galaxies at later epochs (Bromm et al. 2001; Tumlinson et al. 2003). Within the Pop III category, the terms Pop III.1 and Pop III.2 have recently arisen in the literature. Typically, Pop III. 1 connotes the very first stars (i.e., first-generation metalfree stars) that have masses exceeding $\sim 100-300 M_{\odot}$, and that form in $\sim 10^{6} M_{\odot}$ dark matter (DM) minihalos. Pop III.2 stars form in the wake of the radiative and chemodynamic feedback of Pop III.1 supernovae. Pop III.2 are therefore "secondgeneration" stars with metal-free composition, and are thought to have lower stellar masses on average $\left(\sim 10-100 M_{\odot}\right)$ than Pop III.1 stars (Tumlinson et al. 2004; McKee \& Tan 2008; Ohkubo et al. 2009; see, however, Clark et al. 2011 for arguments on Pop III.1 stars having lower stellar masses than Pop III.2 stars). Although Pop III.1 stars were initially thought to form in isolation, producing a single very massive star per minihalo (Abel et al. 2002), this paradigm has lately given way to one where they form mostly in pairs, or systems of even higher multiplicity (Krumholz et al. 2009; Turk et al. 2009; Stacy et al. 2010). 
Recent observations of the galaxy luminosity function (LF) at high redshifts, $z=4-8$ (Bouwens et al. 2010, 2011) indicate that the earliest, faintest galaxy halos make a substantial contribution to reionization, in good agreement with the hypothesis that the first stars are indeed the first ionizing sources. Connecting stellar population models to UV observations of the faint-end galaxy LF is fraught with systematic uncertainties, however, as it requires extrapolation of the observations to even higher redshifts $(z \gtrsim 8)$, and making a number of assumptions with regard to the parameterization of the underlying reionization models. For these reasons, and given the relative insensitivity of present-day $21 \mathrm{~cm}$ data to reionization physics, we focus on constraints from the $\mathrm{CMB}$ in this paper.

It has been shown (Spolyar et al. 2008; Natarajan et al. 2009) that DM could have had a more direct impact on Pop III.1 star formation than simply facilitating the initial baryonic collapse. If DM consists of a new particle that is present in equal numbers to its antiparticle, or if it is indeed its own antiparticle, it will self-annihilate to produce Standard Model (SM) particles such as quarks, photons, and electrons. This is the case for, e.g., weakly interacting massive particles (WIMPs), arguably the most widely studied and natural solution to the DM problem (see, e.g., Jungman et al. 1996; Bergström 2000; Bertone et al. 2005; Bertone 2010). As baryons cool and contract during star formation, they steepen the gravitational potential within the minihalo, drawing even more DM into its center (Freese et al. 2009). The resultant spike in the DM annihilation rate can inject an appreciable amount of energy into the collapsing cloud, halting or delaying star formation (Mapelli et al. 2006; Stasielak et al. 2007; Ripamonti et al. 2007, 2010) and resulting in a cool, partially collapsed object known as a "dark star" (Spolyar et al. 2008; Natarajan et al. 2009). Ongoing annihilation of DM particles in the core of a star can have substantial impacts on its structure and evolution (Salati \& Silk 1989; Fairbairn et al. 2008; Scott et al. 2008, 2009; Iocco 2008; Iocco et al. 2008; Freese et al. 2008a; Spolyar et al. 2009).

As dark stars have significantly different structures and evolutionary histories to "normal" Pop III stars, their ionizing photon outputs differ substantially as well (Yoon et al. 2008), leading to a potentially distinct impact on the process of reionization (Schleicher et al. 2009). The purpose of this paper is to systematically investigate the effects of dark stars on the reionization history of the universe. We begin by discussing dark star formation and evolution in Section 2, and stellar population models including dark stars in Section 3. We give an overview of our reionization models and calculations in Section 4. In Section 5, we give the resulting alternative reionization histories for universes containing dark stellar populations. In Section 6, we show how such IGM ionization histories would impact the measured optical depth to electron scattering in the CMB, drawing limits on dark star populations using the integrated optical depth measured by the Wilkinson Microwave Anistropy Probe seven-year mission (WMAP7), and making predictions for the corresponding constraining power of Planck. We also show the impact of dark stars on the EE polarization anisotropy spectrum of the CMB, and discuss its potential use for constraining dark star populations. We conclude in Section 7.

\section{FORMATION AND EVOLUTION OF DARK STARS}

Assuming that DM self-annihilates, the formation of a dark star relies on some efficient means for bringing the DM into the center of a star. The two processes which may provide this means are gravitational contraction and nuclear scattering.
The first is simply an effect of the changing gravitational potential during the collapse of a baryonic gas cloud (Freese et al. 2008b). As baryons cool and collapse onto the central overdensity, dissipating energy by radiative emission and angular momentum by hydrodynamic and magnetic interactions (which may be enhanced during the formation of the first stars by dynamo effects; Schleicher et al. 2010; Sur et al. 2010), the gravitational potential in the core of the halo steepens. In turn, the steepening potential draws DM into the center of the cloud (despite its inability to actually dissipate energy), resulting in a strongly peaked DM density distribution. This is referred to simply as "gravitational contraction," and is more general than the well-known case of adiabatic contraction because it does not strictly require that the gravitational potential change more slowly than the orbital timescale of individual particles. In the canonical scenario, where a single Pop III.1 star forms at the center of the very first halos, stars begin their lives already in possession of a large reservoir of DM.

The second way for DM to end up in a stellar core relies on it possessing a weak-scale scattering cross-section with nucleons. Such an interaction is characteristic of WIMP DM. Assuming such a cross-section, DM particles passing through a star can lose energy through collisions with stellar nuclei, becoming gravitationally bound to the star (Steigman et al. 1978; Krauss et al. 1985; Press \& Spergel 1985; Gould 1987). This leads to repeat scattering events, eventually removing enough energy that the particle ends up in the stellar core.

Regardless of the path DM follows into a star, the effects are essentially the same. DM annihilation in the core provides an additional energy source alongside nuclear fusion, causing the core to expand and cool. This occurs due to the negative specific heat of a self-gravitating body, and the fact that the DM annihilation rate is decoupled from the nuclear core density. The core expansion leads to a larger, cooler, typically strongly convective stellar object (Spolyar et al. 2008; Scott et al. 2009; Spolyar et al. 2009; Casanellas \& Lopes 2009). In the case of gravitationally contracted DM, the collapse of the forming star is slowed, effectively extending the protostellar phase. The slowdown allows gas accretion to continue longer than it otherwise would, as the onset of radiative feedback is delayed. The resulting object therefore grows more than in the absence of DM (Spolyar et al. 2008; Umeda et al. 2009), leading to masses of the order of $\sim 800-1000 M_{\odot}$. It has been suggested that supermassive objects might even be possible (Freese et al. 2010), though this is strongly constrained by existing data (Zackrisson et al. 2010b).

The degree to which a star affected by DM annihilation resembles either a fully fledged dark star (during the extended protostellar phase) or a main-sequence (MS) object depends on the rate of annihilation in its core. Higher rates of annihilation are required to support larger, more diffuse, protostellar-like structures against further collapse. The evolution of a dark star therefore depends strongly on the rate at which DM is delivered to the stellar core, and how that rate changes over time. Typically, gravitationally contracted DM will be exhausted in a period of $\sim 0.4$ Myr (Spolyar et al. 2009). ${ }^{5}$ Without replenishment via DM capture due to nuclear scattering, dark stars then contract,

\footnotetext{
5 We note, however, that some uncertainty remains over this value, with much shorter timescales suggested by Iocco et al. (2008). Ripamonti et al. (2010) even show that at least in the early stages of the collapse, DM annihilation might in fact help the gas to cool and contract rather than hinder it, by enhancing the formation of $\mathrm{H}_{2}$ molecules; this effect has yet to be taken into account in most dark star modeling.
} 
heat up, and move on to the MS to live their lives as extremely massive Pop III.1 stars.

In the simplest scenario, where the DM halo is homogeneous and spherically symmetric, DM capture by nuclear scattering has been shown not to substantially extend the lifetime of dark stars (Sivertsson \& Gondolo 2011). The impact of more realistic halo distributions on the DM star-crossing rate, and therefore the capture rate, remains to be understood, however (see, e.g., Freese et al. 2010 for the suggestion that particle orbits may even be strongly centrophilic). In principle, if capture rates due to nuclear scattering are high enough, dark stars may exist as cool, diffuse objects for up to $\sim 500 \mathrm{Myr}$ (see, e.g., Zackrisson et al. 2010a, for a discussion of possible dark star lifetimes).

Similarly, the impact of Pop III.1 stars forming as binaries or higher-multiplicity systems is not yet well understood. At some level, the displacement of the collapsing baryonic core(s) from the central DM spike may indeed prevent dark star formation altogether. Alternatively, the fragmentation process may introduce sufficient structure to the phase-space distribution of the DM halo that the findings of Sivertsson \& Gondolo (2011) are circumvented, and dark star lifetimes in fact become longer. In this case, however, the resulting dark stars might be of substantially lower mass than those formed in isolation, due to reduced accretion onto the central object (Peters et al. 2010), shown to be significant for first star formation (Clark et al. 2011). Further review of the structure and evolution of dark stars and the mechanisms for fueling them can be found in Scott (2011).

Given the substantial theoretical uncertainty in predicting the longevity of dark stars, for the purposes of this paper we consider their lifetimes to be a free parameter, to be constrained by $\mathrm{CMB}$ data or other observations of reionization. Although we choose a halo mass threshold at $z=20$ that is designed to allow the formation of a single dark star in the very smallest halos (see Section 4), we do not make any strong assumptions as to the number of dark stars forming in each halo in general, as the reionization formalism described in Section 4 is non-specific as to the multiplicity of star formation.

\section{DARK AND SEMI-DARK STELLAR POPULATIONS}

\subsection{Population Models}

Modeling a stellar population containing dark stars requires careful consideration of the possible evolutionary histories of the dark component. We define different structural forms for dark stars, depending on the degree to which they are dominated by DM: the dark star proper (DSP) and the dark star near the main sequence (DSNMS). The DSP structure occurs when DM annihilation contributes a substantial fraction of the star's energy budget, leading to the large, diffuse, cool objects discussed by Spolyar et al. (2008). These objects lie far to the right of the $\mathrm{H}-\mathrm{R}$ diagram, in the region populated by protostars on their way to the MS. The DSNMS structure occurs when DM contributes only a small amount of the star's total energy budget, and much more closely resembles an MS star. Correspondingly, they lie only slightly to the right of the standard Pop III MS on the H-R diagram.

Of course, the two structures are not entirely distinct; a continuum of stellar structures is possible, parameterized by the amount of energy produced by DM annihilation in the stellar core (as discussed in Section 2). Exactly what structure a dark star exhibits depends on its age (with the DSNMS stage always following the DSP stage) and the rate at which DM is captured and converted into heat. Sustained high rates of capture therefore effectively increase the duration of the DSP phase, but not the DSNMS phase, as the star is supported by DM annihilation in a cool, diffuse configuration until the DM runs out. Correspondingly, sustained moderate capture rates increase the duration of the DSNMS phase, but not the DSP phase, as the star is supported by DM annihilation and nuclear burning, in a configuration that is only slightly cooler than the equivalent MS structure (see, e.g., Scott et al. 2009).

We take a phenomenological definition for the exact demarcation between the DSP and DSNMS phases. The DSP phase includes all structures where capture rates are sufficiently large to keep the star too cool to contribute to reionization $(Q \sim 0$; cooler stars are redder, so more of their luminosity is output at wavelengths longward of the neutral hydrogen ionization threshold). Structures in the DSNMS phase, on the other hand, have small enough WIMP capture rates to be sufficiently hot to make some contribution to reionization. Because the ionizing photon flux for a given stellar mass falls off very abruptly as the relative contribution of DM annihilation to the star's total energy budget is increased, to a first approximation stars in this phase can be modeled as MS objects.

We define three distinct capture scenarios: no capture (NC), meager capture (MC), and extreme capture (EC). NC is the canonical scenario discussed and simulated using a simple varying-index polytropic model by Spolyar et al. (2009): dark stars form by gravitational collapse, and grow to exhibit larger masses than typical Pop III.1 stars because annihilation of gravitationally contracted DM in their cores inhibits the collapse. After the initial (gravitationally contracted) population of DM is exhausted, the star finishes contracting and makes its way to the MS, where it lives like any other $\sim 800 M_{\odot}$ star. In this case, we have a DSP phase with a duration approximately equal to the ages of the stars of Spolyar et al. (2009) on reaching the MS $\left(t_{\mathrm{DSP}} \sim 0.4 \mathrm{Myr}\right.$ for a DM mass of $\left.m_{\chi}=100 \mathrm{GeV}\right)$, followed by a DSNMS phase of duration equal to the standard MS lifetime of an $\sim 800 M_{\odot}$ MS Pop III.1 star $\left(t_{\text {DSNMS }} \sim 1\right.$ Myr; e.g., Schaerer 2002).

In the MC scenario, we have enough DM capture to extend the lifetime $t_{\text {DSNMS }}$ of the DSNMS phase, whereas in the EC scenario we have enough DM capture to instead extend $t_{\mathrm{DSP}}$, the lifetime of the DSP phase. In these two scenarios, we therefore take the lifetimes of the respective phases as free parameters, and keep the lifetime of the other phase fixed at its canonical value in the NC scenario.

In general, the maximum duration of any phase of the lifetime of a star powered by DM capture (either wholly or partially) is limited by the total core hydrogen-burning lifetime of the star, and the self-annihilation time of the DM halo from which the star captures its DM. For stars in the most DM-dominated parts of the DSP phase, the low core temperature and very high capture rate mean that the self-annihilation time is the relevant limit, and the hydrogen-burning time plays little role. For stars in the DSNMS phase, the opposite is true. These limits were calculated and discussed in detail by Zackrisson et al. (2010a). Here we approximately adopt the values $t_{\max }$ of Zackrisson et al. as the upper limits for the durations of the two phases. The lower limits are given by the NC case, which can be seen as a limiting case of both the MC and EC scenarios (although physically, it is of course only continuously linked to the MC case). This gives $0.4 \mathrm{Myr} \leqslant t_{\mathrm{DSP}} \lesssim 500 \mathrm{Myr}$ and $1 \mathrm{Myr} \leqslant t_{\mathrm{DSNMS}} \lesssim 6 \mathrm{Myr}$.

Finally, we also have the dark star mass fraction $f_{\mathrm{DS}}$ as an additional free parameter in all three scenarios (NC, MC, and $\mathrm{EC})$. This describes the fraction of the star-forming baryonic 
mass that initially goes into dark stars rather than normal Pop III stars. We begin with an initial population consisting of a fraction $f_{\text {DS }}$ of dark stars in the DSP phase and a complementary fraction $\left(1-f_{\mathrm{DS}}\right)$ of normal Pop III stars. As the normal Pop III stars finish their starburst (i.e., after $t_{\text {Pop III }}$, which we set to $10 \mathrm{Myr}$ ), they are replaced with Pop II stars. As the dark stars transition from DSP to DSNMS and then eventually die, they are replaced with either normal Pop III stars if the death occurs before the end of the original Pop III starburst, i.e., $t_{\text {DSP }}+t_{\text {DSNMS }}<t_{\text {Pop III }}=10 \mathrm{Myr}$, or directly with Pop II stars if the death occurs after the cessation of the original Pop III starburst, i.e., $t_{\mathrm{DSP}}+t_{\mathrm{DSNMS}} \geqslant t_{\mathrm{Pop} \mathrm{III}}=10 \mathrm{Myr}$. In the case where the dark stars die and are replaced by a new population of Pop III stars, these Pop III stars later also die and are replaced by Pop II stars at $t=t_{\text {Pop III }}=10 \mathrm{Myr}$ after the beginning of star formation, just like their counterparts in the complementary fraction $\left(1-f_{\mathrm{DS}}\right)$ of normal Pop III stars present from the beginning of the calculation.

For ease of reference, the full set of scenarios and parameters with which we compute reionization histories is given in Table 1.

For some combinations of parameters (large $f_{\mathrm{DS}}$ and long $\left.t_{\mathrm{DSP}}\right)$, the astute reader will have realized that our population models contain either very few or no normal Pop III stars. Constructing a consistent picture of the chemical evolution of the universe in these cases becomes somewhat more problematic than in the standard situation, where supernovae produced by the deaths of the original Pop III stars provide the necessary chemical enrichment of gas to facilitate the formation of Pop II stars. Stars as heavy as the $800 M_{\odot}$ dark stars we consider here are typically expected to collapse directly to black holes (e.g., Umeda et al. 2009), producing very few metals. Because only stars in the mass range up to $260 M_{\odot}$ produce metals (Heger \& Woosley 2002; Venkatesan \& Truran 2003), this constrains the mass range of a primordial stellar population that must necessarily seed the conditions for Pop II star formation (and this is used to justify the Pop III mass function we consider below). Moreover, the yields of heavy elements from supernovae from the first stars as well as the true distribution of metallicities in the very first Pop II stars are currently not well constrained, so it is conceivable that a small number of highly efficient Pop III stars could provide the bulk of chemical enrichment necessary to allow the transition to Pop II (and such a Pop II may have begun somewhat later in some locations, and with a somewhat smaller metallicity than is considered typical). In any case, stars as massive as $800 M_{\odot}$ operate very close to the Eddington luminosity, and so are expected to exhibit very strong stellar winds and experience numerous mass-loss events. Although mass loss from metal-poor or metal-free stars is expected to be substantially reduced in comparison to that from their metal-rich cousins, depending on the rotational and convective properties of the first stars, the material blown off from such objects may well be sufficiently processed to also contribute to the chemical enrichment of the universe.

\subsection{Ionizing Photon Fluxes}

At each time step of our reionization calculation, we calculate the weighted-average, mass-normalized, hydrogen-ionizing photon output of the combined stellar population as

$$
Q_{\mathrm{tot}}(t)=f_{\mathrm{DS}} Q_{\mathrm{DS}}(t)+\left(1-f_{\mathrm{DS}}\right) Q_{\mathrm{normal}}(t) .
$$

Here, $Q_{\mathrm{DS}}(t)$ refers to the ionizing photon output per unit mass of stars in the population originally consisting of dark
Table 1

Scenarios, Parameters, Redshifts of H I Reionization and Integrated Optical Depths to Last Scattering for Stellar Populations with Dark Stars

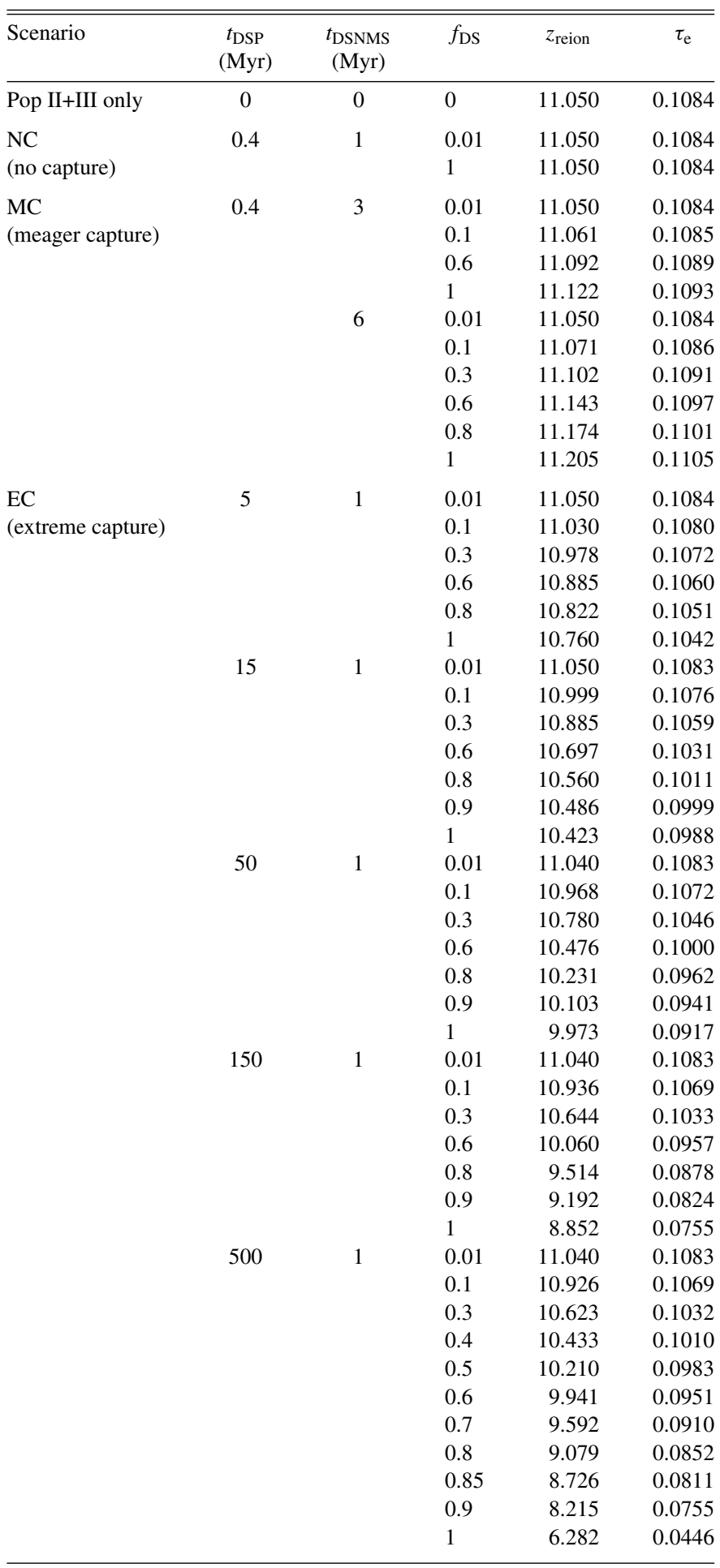

Notes. Optical depths include contributions from He II, He III, and residual electron fraction after recombination. See the text for details.

stars. Depending on the time $t$ in question, and the values of the lifetime parameters $t_{\mathrm{DSP}}$ and $t_{\mathrm{DSNMS}}$, this may be equal to either $Q_{\mathrm{DSP}}, Q_{\mathrm{DSNMS}}, Q_{\mathrm{Pop} \mathrm{II}}$, or $Q_{\mathrm{Pop} \mathrm{III}} \cdot Q_{\text {normal }}(t)$ refers to the population originally consisting of normal Pop III stars, and is equal to $Q_{\text {Pop III }}$ for $t<10 \mathrm{Myr}$, and $Q_{\text {Pop II }}$ for $t \geqslant 10 \mathrm{Myr}$. 
Table 2

Ionizing Photon Fluxes, with $Q_{\mathrm{H}}$ Values High Enough to Contribute to Reionization (cf. Table 1 in Tumlinson et al. 2004) Marked in Bold

\begin{tabular}{lcccc}
\hline \hline DM Mass & $\begin{array}{c}\text { Age } \\
(\mathrm{yr})\end{array}$ & $\begin{array}{c}\text { Stellar } \\
\text { Mass }\left(M_{\odot}\right)\end{array}$ & $\begin{array}{c}Q_{\mathrm{H}} \\
\left(\mathrm{s}^{-1}\right)\end{array}$ & $\begin{array}{c}Q_{\mathrm{H}} \\
\left(\mathrm{s}^{-1} M_{\odot}^{-1}\right)\end{array}$ \\
\hline $1 \mathrm{GeV}$ & $3.1 \times 10^{5}$ & 756 & $1.05 \times 10^{44}$ & $1.38 \times 10^{41}$ \\
$1 \mathrm{GeV}$ & $3.3 \times 10^{5}$ & 793 & $\mathbf{4 . 2 6} \times 10^{49}$ & $\mathbf{5 . 3 7} \times 10^{46}$ \\
$1 \mathrm{GeV}$ & $4.6 \times 10^{5}$ & 824 & $\mathbf{5 . 3 7} \times 10^{50}$ & $\mathbf{6 . 5 2} \times 10^{47}$ \\
$100 \mathrm{GeV}$ & $3.0 \times 10^{5}$ & 716 & $6.28 \times 10^{47}$ & $8.78 \times 10^{44}$ \\
$100 \mathrm{GeV}$ & $3.9 \times 10^{5}$ & $779^{\mathrm{a}}$ & $\mathbf{4 . 9 7} \times 10^{50}$ & $\mathbf{6 . 3 8} \times 10^{47}$ \\
$100 \mathrm{GeV}$ & $4.1 \times 10^{5}$ & $787^{\mathrm{b}}$ & $\mathbf{5 . 2 0} \times 10^{50}$ & $\mathbf{6 . 6 1} \times 10^{47}$ \\
$10 \mathrm{TeV}$ & $0.9 \times 10^{5}$ & 327 & $3.40 \times 10^{43}$ & $1.04 \times 10^{41}$ \\
$10 \mathrm{TeV}$ & $2.7 \times 10^{5}$ & 553 & $\mathbf{3 . 2 7} \times 10^{50}$ & $\mathbf{5 . 9 2} \times 10^{47}$ \\
Normal Pop II & & $1-100$ & & $\mathbf{7 . 7 6} \times 10^{46}$ \\
Normal Pop III & & $10-140$ & & $\mathbf{4 . 3 0} \times 10^{47}$ \\
\hline
\end{tabular}

Notes.

${ }^{a}$ This case corresponds to the NC scenario considered in this work, and does not include any capture by nuclear scattering. All other entries in this table, including the model used for the MC and EC scenarios, correspond to models where nuclear scattering provides a similar amount of power to DM obtained by gravitational contraction (see Spolyar et al. 2009 for details).

$\mathrm{b}$ This case is used in calculations for the EC and MC scenarios considered in this work.

Explicitly, if we designate $t_{0}$ as the time elapsed since the onset of star formation (DS and/or Pop III), and define the specific averaged $Q_{\mathrm{H}}$ factors

$$
\begin{aligned}
Q_{\mathrm{tot}, \mathrm{DSP}+\mathrm{P} 3} & =\left(1-f_{\mathrm{DS}}\right) Q_{\mathrm{Pop} \mathrm{III}} \\
Q_{\mathrm{tot}, \mathrm{DSP}+\mathrm{P} 2} & =\left(1-f_{\mathrm{DS}}\right) Q_{\mathrm{Pop} \mathrm{II}} \\
Q_{\mathrm{tot}, \mathrm{DSNMS}+\mathrm{P} 3} & =f_{\mathrm{DS}} Q_{\mathrm{DSNMS}}+\left(1-f_{\mathrm{DS}}\right) Q_{\mathrm{Pop} \mathrm{III}} \\
Q_{\mathrm{tot}, \mathrm{DSNMS}+\mathrm{P} 2} & =f_{\mathrm{DS}} Q_{\mathrm{DSNMS}}+\left(1-f_{\mathrm{DS}}\right) Q_{\mathrm{Pop} \mathrm{II}},
\end{aligned}
$$

we have three possible scenarios, depending on the relative values of the lifetime parameters $t_{\mathrm{DSP}}, t_{\mathrm{DSNMS}}$, and $t_{\mathrm{Pop} \mathrm{III}}$ :

1. $t_{\mathrm{DSP}}+t_{\mathrm{DSNMS}}<t_{\mathrm{Pop} \mathrm{III}}$

(a) $Q_{\mathrm{H}}=Q_{\text {tot,DSP }+\mathrm{P} 3}$, duration $t_{\mathrm{DSP}}$

(b) $Q_{\mathrm{H}}=Q_{\text {tot,DSNMS+P3}}$, duration $t_{\mathrm{DSNMS}}$

(c) $Q_{\mathrm{H}}=Q_{\text {Pop III }}$, duration $t_{\mathrm{Pop} \mathrm{III}}-\left(t_{\mathrm{DSNMS}}+t_{\mathrm{DSP}}\right)$

(d) $Q_{\mathrm{H}}=Q_{\text {Pop II }}$, duration $t_{0}-t_{\text {Pop III }}$

2. $t_{\mathrm{DSP}}<t_{\mathrm{Pop} \mathrm{III}}$ and $t_{\mathrm{DSP}}+t_{\mathrm{DSNMS}}>t_{\mathrm{Pop} \mathrm{III}}$

(a) $Q_{\mathrm{H}}=Q_{\mathrm{tot}, \mathrm{DSP}+\mathrm{P} 3}$, duration $t_{\mathrm{DSP}}$

(b) $Q_{\mathrm{H}}=Q_{\text {tot,DSNMS+P3 }}$, duration $t_{\text {Pop III }}-t_{\text {DSP }}$

(c) $Q_{\mathrm{H}}=Q_{\mathrm{tot}, \mathrm{DSNMS}+\mathrm{P} 2}$, duration $t_{\mathrm{DSNMS}}-\left(t_{\mathrm{Pop} \mathrm{III}}-t_{\mathrm{DSP}}\right)$

(d) $Q_{\mathrm{H}}=Q_{\mathrm{Pop} \mathrm{II}}$, duration $t_{0}-\left(t_{\mathrm{DSP}}+t_{\mathrm{DSNMS}}\right)$

3. $t_{\mathrm{DSP}}>t_{\mathrm{Pop} \mathrm{III}}$
(a) $Q_{\mathrm{H}}=Q_{\mathrm{tot}, \mathrm{DSP}+\mathrm{P} 3}$, duration $t_{\mathrm{Pop} \mathrm{III}}$
(b) $Q_{\mathrm{H}}=Q_{\text {tot,DSP+P2 }}$, duration $t_{\mathrm{DSP}}-t_{\mathrm{Pop} \mathrm{III}}$
(c) $Q_{\mathrm{H}}=Q_{\text {tot,DSNMS+P2 }}$, duration $t_{\mathrm{DSNMS}}$
(d) $Q_{\mathrm{H}}=Q_{\text {Pop II }}$, duration $t_{0}-\left(t_{\mathrm{DSP}}+t_{\mathrm{DSNMS}}\right)$.

For all scenarios, and in Equations (2) and (3) above, we assume $Q_{\text {DSP }}=0$ during the DSP phase.

We calculate ionizing photon fluxes during the DSNMS phase using model dark star atmospheres computed with TLUSTY (Hubeny \& Lanz 1995), as described in Zackrisson et al. (2010a). Hydrogen-ionizing photon fluxes for some example dark star models computed by Spolyar et al. (2009) are shown in Table 2. Here we display $Q_{\mathrm{H}}$ values for three different stellar models, computed assuming three different DM masses. We show snapshots of $Q_{\mathrm{H}}$ at different times in the respective models' evolution: larger stellar masses correspond to later models, as dark stars gradually accrete more matter. We do not show $Q_{\mathrm{H}}$ values for earlier times in the simulations (corresponding to the main part of the DSP phase), as the ionizing fluxes of the earliest (lowest-mass) snapshots shown in Table 2 are already too low to be significant for reionization. As is to be expected, ionizing photon fluxes increase with time and stellar mass as the dark stars become hotter, more compact and luminous as they move from DSP to DSNMS, and finally, to the zero-age MS.

For a fixed initial DM density, larger DM masses lead to slightly decreased energy production in the stellar core (due to the decreased number density implied by a constant mass density). This can be seen in the lower final mass of the $m_{\chi}=1$ $\mathrm{TeV}$ model in Table 2, where DM annihilation has extended the accretion phase during the DSP less than it would if the DM mass were smaller (see Spolyar et al. 2009 for more details), resulting in a reduced $Q_{\mathrm{H}}$ during the DSNMS phase. In general, however, the mass of the DM particle has only a weak impact on the phenomenology of dark stars. For the remainder of this paper, we focus on the example case $m_{\chi}=100 \mathrm{GeV}$.

For the NC scenario, we use the final, $779 M_{\odot}, m_{\chi}=$ $100 \mathrm{GeV}$ stellar model of Spolyar et al. (2009), which was computed without including any capture of WIMPs via nuclear scattering. When calculating ionizing fluxes during the DSNMS phase in the MC and EC scenarios, we instead use the corresponding $787 M_{\odot}, m_{\chi}=100 \mathrm{GeV}$ model, which included a small amount of DM capture by nuclear scattering (see Spolyar et al. for details). In practice, there is very little difference between the models of Spolyar et al. (2009) with and without the very small amount of capture they included.

The ionizing photon fluxes of our canonical Pop II and III populations are also given in Table 2.

\section{REIONIZATION CALCULATIONS}

We use the semianalytic reionization model in Venkatesan et al. (2003) for a $\Lambda$ CDM cosmology. The growth of ionized regions is tracked by a Press-Schechter formalism in 
combination with numerical solutions for the growth of individual ionization fronts. We take our cosmological parameter set from the latest WMAP7 results (Larson et al. 2011). ${ }^{6} \mathrm{We}$ assume that the fraction of baryons forming stars in each halo is $f_{\star}=0.05$, and that the escape fraction of $\mathrm{H}$ II ionizing radiation from halos is $f_{\text {esc }}=0.1$. Theoretical calculations, both analytical and from numerical simulations, as well as observations of star-forming galaxies in the local and high- $z$ universe, indicate that $f_{\star}=0.01-0.1$, and $f_{\text {esc }}=0.01-0.2$ (Venkatesan et al. 2003 and references therein).

We allow star formation in all halos of virial temperature \375 K (rather than $10^{4} \mathrm{~K}$ as in Venkatesan et al. 2003, but similar to $10^{3} \mathrm{~K}$ in Tumlinson et al. 2004) starting at $z=20$. This ensures that in our cases where $f_{\mathrm{DS}}=1$, there are sufficient baryons in a $10^{6} M_{\odot}$ DM halo at $z \sim 20$ for our adopted star formation efficiencies to form a single $800 M_{\odot}$ dark star. This relies on the assumption that halos with virial temperatures $\lesssim 10^{4} \mathrm{~K}$ can cool effectively using, e.g., $\mathrm{H}_{2}$ or other pathways (Abel et al. 2002; Haiman \& Holder 2003). ${ }^{7}$ Increasing the virial temperature threshold (i.e., the minimum mass scale of collapsing halos) to values higher than our choice here will delay reionization correspondingly. As the most interesting constraints we produce are based on dark-starinduced delays to reionization, this choice is a conservative one.

We assume Pop III (metal-free) stars can form starting at $z=$ 20, in a Salpeter-slope initial mass function (IMF) spanning the range $10-140 M_{\odot}$. This Pop III IMF $^{8}$ is one lacking in lowmass stars, and was motivated by ionization constraints and observations of abundance trends in metal-poor Galactic halo stars (Tumlinson et al. 2004). It has a mass-normalized ionizing photon flux about a factor of three smaller than the ionizing flux of an IMF containing very massive stars $\left(10^{2}-10^{3} \mathrm{M}_{\odot}\right.$; Bromm et al. 2001). We assume that the Pop III phase lasts for $10 \mathrm{Myr}$ in each halo, in agreement with the duration of metal-free star formation calculated from numerical simulations of halo self-enrichment (Wada \& Venkatesan 2003), and of interhalo enrichment (Bromm et al. 2003; Tumlinson et al. 2004). Subsequent to $10 \mathrm{Myr}$ after the onset of star formation in each halo, the ionizing spectrum is switched to a representative example of Pop II stars in a Salpeter-slope IMF in the mass range $1-100 M_{\odot}$ with metallicity $Z=0.001$ (Leitherer et al. 1995).

It is possible that Pop II star formation need not always follow that of Pop III; both populations could form simultaneously in separate gas clouds within individual halos, due to inhomogeneous metal enrichment. However, this is most likely to happen in more massive halos, as smaller halos will experience rapid self-enrichment or lose their gas entirely after the first Pop III supernovae. Massive halos are also more likely to be made up of smaller halos with disparate chemical evolution histories (see

\footnotetext{
6 We used the WMAP7 cosmological parameter set at: http://lambda.gs-fc. nasa.gov/product/map/current/params/lcdm_sz_lens_wmap7.cfm

7 The assumption that each new halo forms metal-free stars breaks down at $z \sim 8-9$, when the earliest small halos, presumably metal-enriched from their first episodes of Pop III star formation, begin to coalesce into larger objects. Most of the scenarios considered here reionize earlier than this; those that do not would arguably not follow the standard timeline of chemical enrichment anyway, as the bulk of baryons would be tied up in long-lived DSP-phase dark stars at redshifts $z \gtrsim 9$, leading to some delay in the chemical evolution of the universe.

8 The Pop III IMF is independent of the presence of dark stars in our formalism, and is not designed to reflect the late stages of dark star evolution in any way. As noted earlier, dark stars die at the end of the DSNMS phase, which is effectively their MS.
}

footnote 7). Such massive halos are more common at $z \sim 7-9$, when the universe is 0.5 Gyr old. This cosmic age is substantially larger than the $10 \mathrm{Myr}$ self-enrichment timescales of halos discussed above, over which the conditions to form Pop III are lost. Additionally, the gain in hydrogen-ionizing photon production from Pop III relative to Pop II is a factor of the order of 0.6 up to a few (Tumlinson et al. 2004). When one considers that this could occur only in larger halos for at most $10 \mathrm{Myr}$ (at a cosmic age of $0.5-1 \mathrm{Gyr}$ ), the impact on reionization should be relatively small. We have confirmed in separate calculations for such a scenario that this is the case. We therefore do not consider simultaneous Pop III and Pop II star formation within the same halo in our models.

Note that in our formalism for using weighted-average ionizing photon fluxes from Pop III, Pop II, and dark stars, at each cosmological epoch, we count the time-appropriate $Q_{\mathrm{H}}$ at that epoch arising from all star-forming halos that have collapsed over a range of redshifts starting at $z=20$. Reionization is defined as the overlap of individual ionized regions of $\mathrm{HII}$, when its IGM volume filling factor equals unity (Venkatesan et al. 2003).

\section{REIONIZATION HISTORY OF A UNIVERSE CONTAINING DARK STARS}

In the NC scenario, where dark stars are fueled by gravitationally contracted DM only, we find that the reionization history of the universe is effectively identical for all values of the dark star fraction $f_{\mathrm{DS}}$ (Table 1$)$. This is despite the fact that during the first $0.4 \mathrm{Myr}$ of star formation, the dark stellar population contributes nothing to reionization, reducing $Q_{\text {tot }}$ to

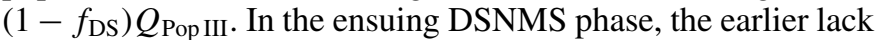
of ionizing photons is compensated for by an excess relative to the canonical situation (where $f_{\mathrm{DS}}=0$ and there are no dark stars, only the normal Pop III followed by Pop II), because $Q_{\text {DSNMS }}>Q_{\text {Pop III. }}$ Although the net impacts of these two effects on the redshift of reionization cancel, one might expect a difference in the time evolution of the ionization fraction relative to the simple Pop III+II case; due to the short durations of the DSP and DSNMS phases in the NC scenario, this effect is too small to notice, however.

In Figure 1, we show ionization histories in the EC scenario, for various combinations of $f_{\mathrm{DS}}$ and the DSP lifetime $t_{\mathrm{DSP}}$. Here we see a marked effect on reionization, with populations containing large numbers of dark stars and/or relatively longlived ones resulting in substantially delayed reionization. As expected, the larger the values of $f_{\mathrm{DS}}$ and $t_{\mathrm{DSP}}$, the larger the effect. For larger $f_{\mathrm{DS}}$ and $t_{\mathrm{DSP}}, z_{\text {reion }}$ occurs later, as $Q_{\text {tot }}$ remains at lower values for longer periods of time during the earliest part of the star formation history in each halo. For longer delays, reionization occurs more quickly as IGM ionization becomes able to build up more quickly. This is a result of two effects: the main sources of ionizing photons turning on at lower redshifts, and the fact that the IGM density decreases rapidly with decreasing redshift, leading to increasing IGM recombination timescales at later cosmic times. This explains why the rapidity of reionization becomes more pronounced with increasing $t_{\mathrm{DSP}}$ than with increasing $f_{\mathrm{DS}}$.

Comparing our results in Table 1 with the constraints from WMAP7, we find that a few of our cases can be immediately ruled out by simply having too low a value of $\tau_{\mathrm{e}}$, the optical depth to electron scattering, or $z_{\text {reion }}$. The global fit to WMAP7 and other cosmological data (Komatsu et al. 2011) implies that the integrated optical depth back to the surface of last scattering (i.e., 

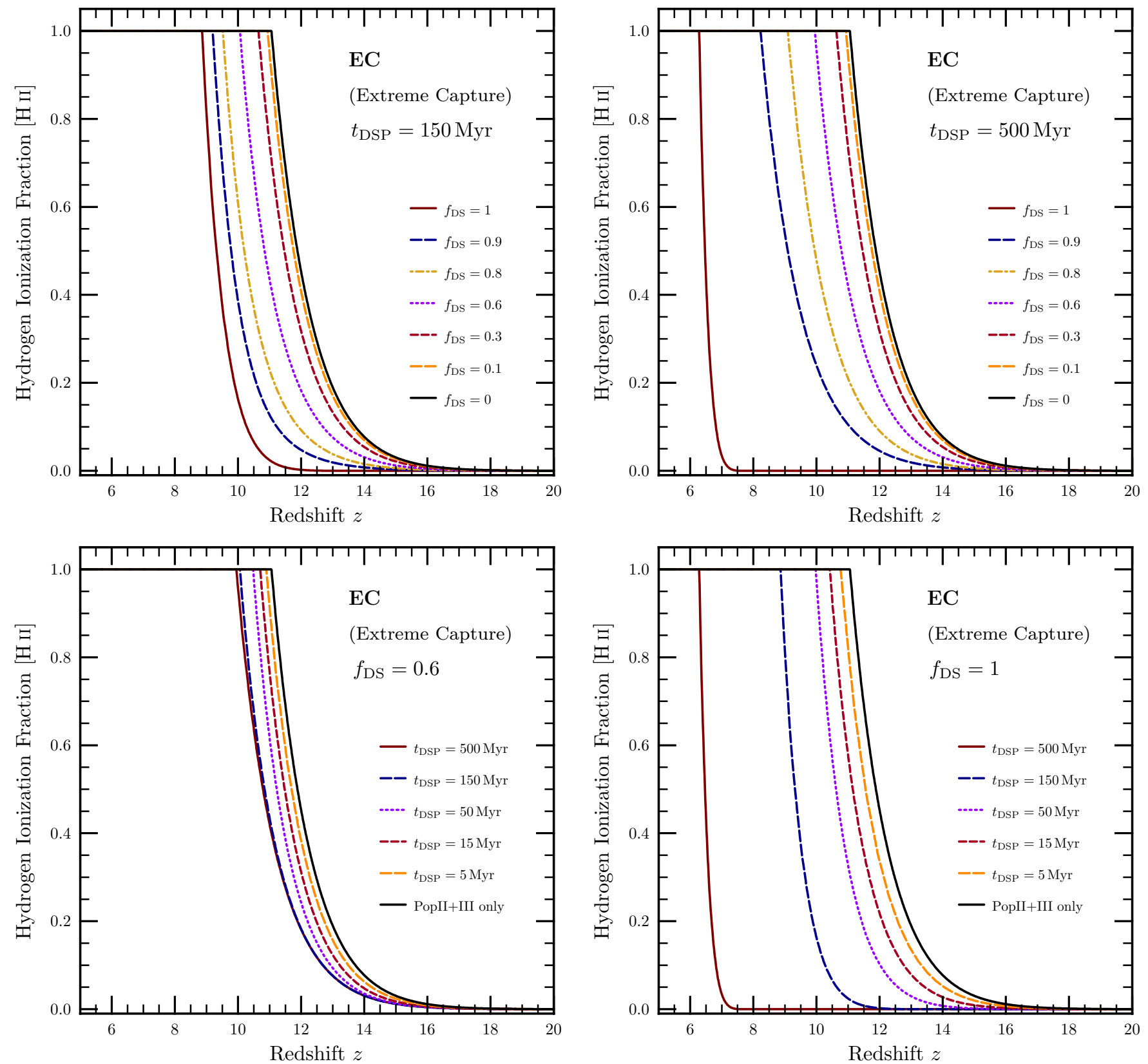

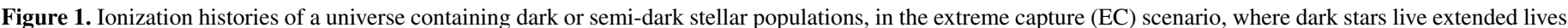

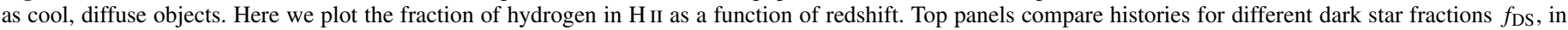

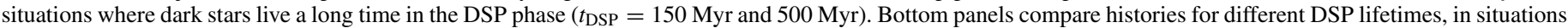

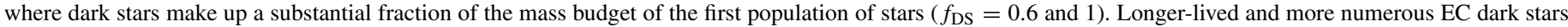

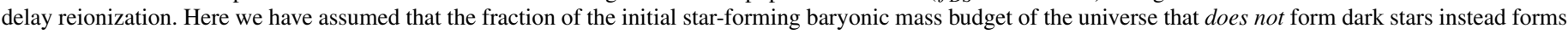

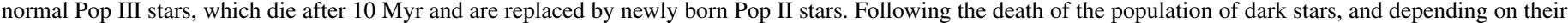

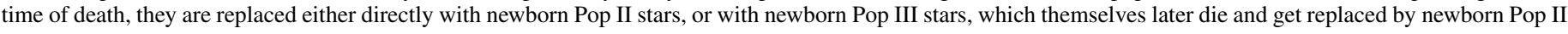
stars.

(A color version of this figure is available in the online journal.)

recombination at $z \sim 1090$ ), is $\tau_{\mathrm{e}} \sim 0.088 \pm 0.014$. For a simple step-function reionization history, this is $z_{\text {reion }}=10.6 \pm 1.2$. A direct comparison with Table 1 would in principle rule out all cases with $\tau_{\mathrm{e}} \lesssim 0.074$, or with $z_{\text {reion }} \lesssim 9$ 9.4. Note that the $\tau_{\mathrm{e}}$ constraint is more direct, and that limits based on $z_{\text {reion }}$ are only approximate owing to the WMAP7 assumption of a simplified reionization history (step-function ionization at fixed $\left.z_{\text {reion }}\right)$. Our models are more realistic and track the details of reionization with variations in galaxy halo masses and astrophysical parameters, so that our derived $z_{\text {reion }}$ and that from
WMAP7 may not be directly comparable. Thus, comparing the values of $\tau_{\mathrm{e}}$ from Table 1 to the WMAP7 limits, we see that the $\mathrm{EC}$ case with $t_{\mathrm{DSP}}=500 \mathrm{Myr}$ and $f_{\mathrm{DS}}=1$ is ruled out. More EC cases, e.g., $t_{\mathrm{DSP}}=150 \mathrm{Myr}$ and $f_{\mathrm{DS}}=0.9-1$, or $t_{\mathrm{DSP}}=500 \mathrm{Myr}$ and $f_{\mathrm{DS}} \gtrsim 0.75$, would be ruled out at face value if we were to compare directly with the WMAP7 limit that $z_{\text {reion }}=9.4-11.8$. Similarly, one might also conclude that many models with low $t_{\mathrm{DSP}}$ and $f_{\mathrm{DS}}$ are ruled out for producing $z_{\text {reion }}$ and $\tau_{\mathrm{e}}$ exceeding the upper limit of the WMAP7 error band. While this is indeed true when $f_{\star} f_{\text {esc }}=0.005$ as we 

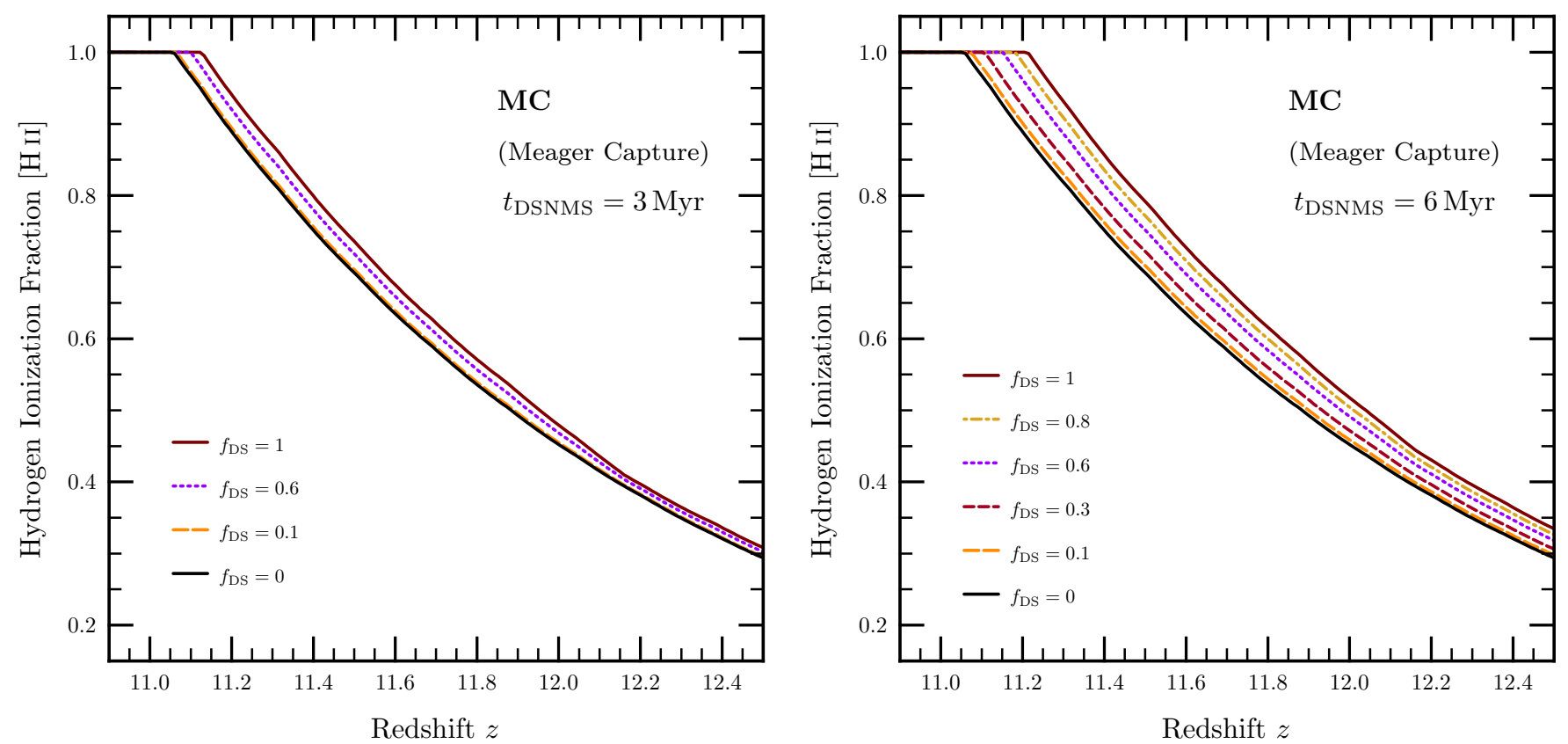

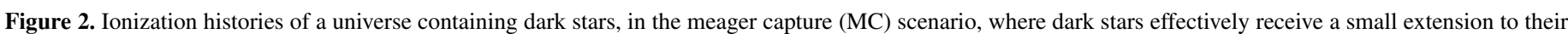

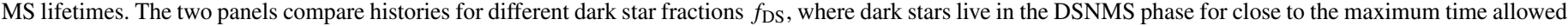

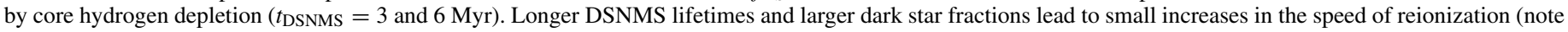
the zoomed axes relative to Figure 1). Non-dark aspects of the calculations are as described for Figure 1.

(A color version of this figure is available in the online journal.)

assume here, such limits are not really robust to variations in astrophysical parameters. We discuss integrated optical depths and corresponding constraints in more detail in the following section.

For the MC scenario, the effects are less dramatic; in Figure 2 we show a zoomed-in section of the full history of reionization, for a few combinations of $f_{\mathrm{DS}}$ and the DSNMS lifetime $t_{\mathrm{DSNMS}}$. Here, increasing $f_{\mathrm{DS}}$ and $t_{\mathrm{DSNMS}}$ results in progressively earlier reionization, the exact opposite trend relative to the EC cases. This is because $Q_{\text {DSNMS }}>Q_{\text {Pop III }}$, so extending the DSNMS phase and increasing the fraction of baryons contained in it causes reionization to happen more quickly than with only a normal Pop III IMF. Again, as expected the effects increase with increasing $f_{\mathrm{DS}}$ and $t_{\mathrm{DSNMS}}$. The reason the MC scenario has a much smaller effect on reionization than the EC scenario is that its duration is already much more strongly constrained, in this case by the fusion-burning timescale of core hydrogen during the DSNMS phase.

In Figure 3, we show the impact of varying the product of the astrophysical parameters $f_{\star}$ and $f_{\text {esc }}$ from our canonical value of $f_{\star} f_{\text {esc }}=0.005$ to the extreme values of $0.02\left(f_{\star}=\right.$ $\left.0.1, f_{\text {esc }}=0.2\right)$ or $10^{-4}\left(f_{\star}=f_{\text {esc }}=0.01\right)$. We give the resulting reionization histories both for a standard Pop III without dark stars, and for an EC example with $f_{\mathrm{DS}}=1, t_{\mathrm{DSP}}=$ $150 \mathrm{Myr}$. Here, we see that within this range of astrophysical uncertainties, a large range of reionization histories is possible. Indeed, in the most extreme cases, dark stars have a similar magnitude effect as the variation of astrophysical parameters. This degeneracy is unfortunate, but not unexpected; substantial uncertainty exists in reionization models at present, even before introducing the possibility of stellar populations including dark stars. Hearteningly, however, the impact of the astrophysical uncertainties is reduced in situations where dark stars play a significant role, as can be seen by comparing the two panels of Figure 3.
The results we present here agree broadly with those of Schleicher et al. (2009), but the correspondence is not immediately obvious. Schleicher et al. considered reionization from "MS-dominated" (main sequence), and "CD" (capture dominated) dark stars, roughly corresponding to our own MC and EC scenarios, respectively. They investigated the case where $f_{\mathrm{DS}}=1$, showing that the higher ionizing photon fluxes of the DSNMS phase hasten reionization, whereas the lower fluxes of the DSP phase delay it. This is in good agreement with what we show here, and earlier predictions by Yoon et al. (2008). Where we differ from Schleicher et al.'s analysis is in our atmospheric modeling (we use actual model atmospheres rather than blackbody spectra), and in the details of our population modeling.

Here we carefully treat the allowed lifetimes of the different dark star phases, taking into account existing limits from the timescales of core hydrogen burning and self-annihilation of the DM halos surrounding dark stars. Schleicher et al. (2009) adopted the ionizing photon fluxes of Yoon et al. (2008), which accounted for the limit from core hydrogen burning, but not from halo self-annihilation or disruption (although both did at least acknowledge that this might be a concern). Many of these lifetimes we now know to be theoretically inaccessible due to self-annihilation constraints (see the paragraph discussing viable ranges of $t_{\mathrm{DSP}}$ and $t_{\mathrm{DSNMS}}$ in Section 3.1). Many of the models Schleicher et al. considered were accordingly ruled out by the redshifts of reionization or integrated optical depths. Schleicher et al. (2009) also allowed only one or the other of the DSP or DSNMS phases in their calculations, whereas we include both self-consistently.

Schleicher et al. (2009) went on to investigate whether the effects of dark stars can be compensated for by more complicated reionization histories, varying assorted astrophysical parameters and introducing a second period of recombination followed by a late starburst, leading to a two-stage reionization history. Here 

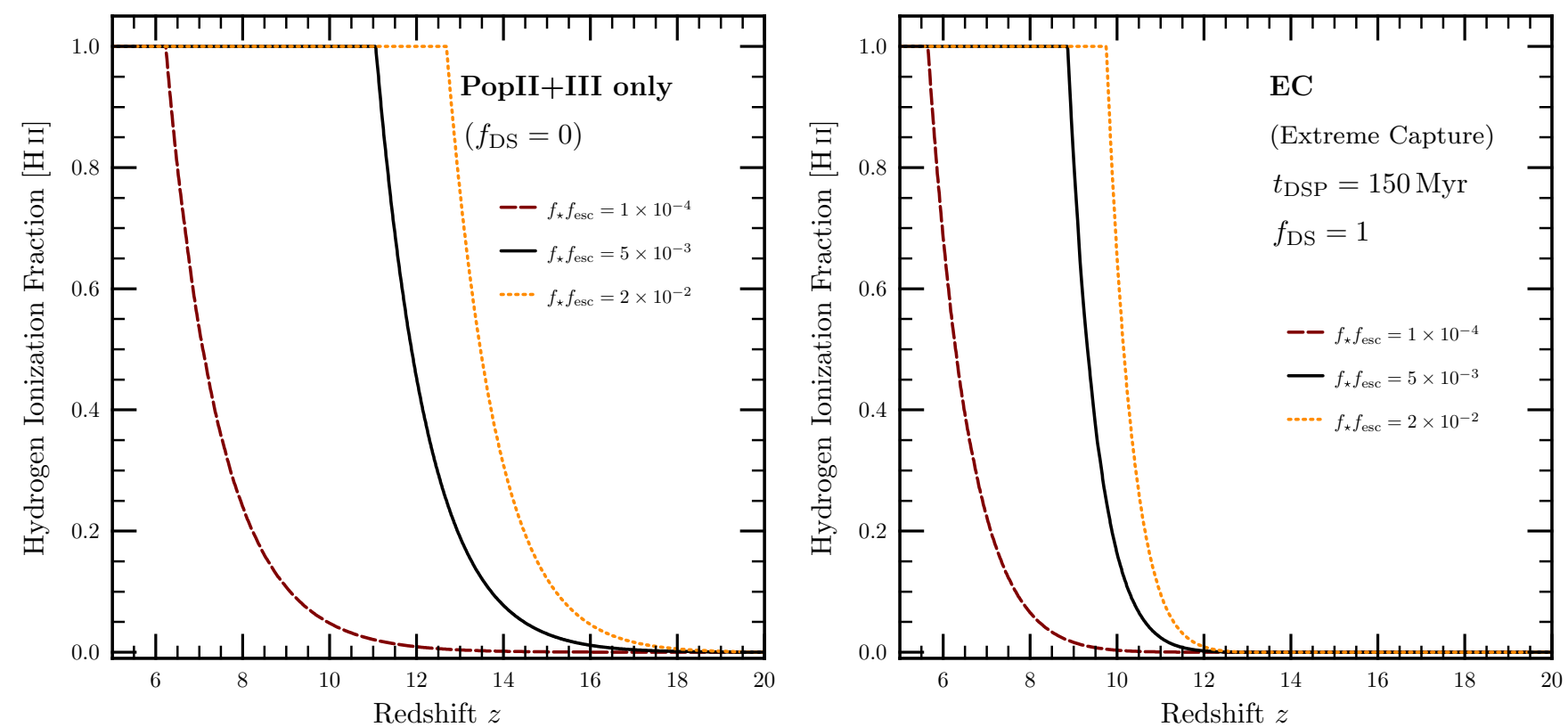

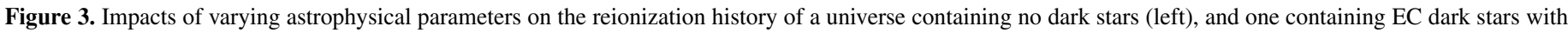

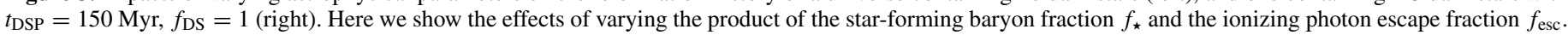

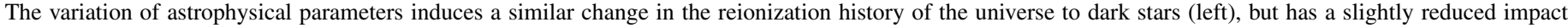

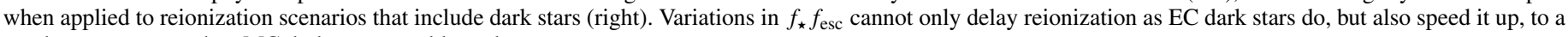
much greater extent than MC dark stars are able to do.

(A color version of this figure is available in the online journal.)

we have shown that although dark stars can have a significant impact on the reionization history of the universe, they need not necessarily. Even in cases with the most extreme effects (e.g., EC scenarios with $f_{\mathrm{DS}}=1, t_{\mathrm{DSP}}>100 \mathrm{Myr}$ ), Figure 3 shows that ad hoc scenarios like those considered by Schleicher et al. are not necessary to reconcile dark stars with reionization constraints; a simple increase in $f_{\star} f_{\text {esc }}$ does the job quite well enough.

\section{IMPACTS ON THE COSMIC MICROWAVE BACKGROUND}

\subsection{Electron-scattering Optical Depths}

Following Shull \& Venkatesan (2008), for each of our reionization histories we calculate the optical depth from the present day to a redshift $z$ due to Thomson scattering as

$$
\tau_{\mathrm{e}}(z)=\frac{c}{H_{0}} \int_{0}^{z} \frac{n_{\mathrm{e}}(z) \sigma_{\mathrm{T}}}{(1+z)\left[\Omega_{\mathrm{m}}(1+z)^{3}+\Omega_{\Lambda}\right]^{1 / 2}} d z,
$$

where

$n_{\mathrm{e}}(z)=\frac{3 \Omega_{\mathrm{b}} H_{0}^{2}}{8 \pi G m_{\mathrm{H}}}(1+z)^{3}\left[X f_{\mathrm{HII}}(z)+\frac{Y}{4}\left\{f_{\mathrm{He} \mathrm{II}}(z)+2 f_{\mathrm{He} \mathrm{III}}(z)\right\}\right]$

is the number density of free electrons. Here, $H_{0}, \Omega_{\mathrm{m}}, \Omega_{\Lambda}$, and $\Omega_{\mathrm{m}}$ are the present-day values of the Hubble constant, mass fraction, baryon fraction, and dark energy fraction of the critical density of the universe, respectively. The electron-photon Thomson-scattering cross-section is given by $\sigma_{\mathrm{T}}$, the mass of hydrogen by $m_{\mathrm{H}}$, the primordial hydrogen mass fraction by $X$, and the primordial helium fraction by $Y \approx 1-X$. The ionization fractions $f_{\mathrm{HII}}, f_{\mathrm{He}}$, and $f_{\mathrm{He} \text { III }}$ refer to the fraction by number of hydrogen or helium atoms, respectively, in the ionization states $\mathrm{H}$ II, He II, and He III. We assume that the number of electrons provided by ionization from $\mathrm{He}$ I to $\mathrm{He}$ II directly tracks hydrogen ionization (i.e., $f_{\mathrm{He} \text { II }}+f_{\mathrm{He} \text { III }}=f_{\mathrm{HII}}$ ), leading to

$$
n_{\mathrm{e}}(z)=\frac{3 \Omega_{\mathrm{b}} H_{0}^{2}}{8 \pi G m_{\mathrm{H}}}(1+z)^{3}\left[\left(1-\frac{3 Y}{4}\right) f_{\mathrm{HII}}(z)+\frac{Y}{4} f_{\mathrm{He} \mathrm{III}}(z)\right] .
$$

We assume a simple step-function ionization model for He III, with $f_{\mathrm{He} \mathrm{III}}(z>3)=0$ and $f_{\mathrm{He} \mathrm{III}}(z \leqslant 3)=1$. We also assume a residual electron fraction from recombination, present even before reionization at the level of $x_{\mathrm{e}}=2.1 \times 10^{-4}$. This number comes from recombination modeling in CAMB (Section 6.2). For both the optical depths based on Equation (6) and CAMB calculations, we use the same values for cosmological parameters as in our reionization calculations, coming from WMAP seven-year results (Larson et al. 2011). We give optical depths integrated up to the surface of last scattering $(z \sim 1090)$ for each of our parameter combinations in Table 1.

In Figure 4, we show the evolution of optical depth corresponding to the ionization histories detailed in Figure 1. As might be expected from the ionization curves, longer-lived and more numerous EC dark stars result in smaller electronscattering optical depths, as they reionize the universe later. The resulting integrated optical depths across the entire EC parameter space are summarized in Figure 5, where we plot $\tau_{\mathrm{e}}$ as a continuous function of $t_{\mathrm{DSP}}$ and $f_{\mathrm{DS}}$.

Similarly, we show a zoomed-in section of the optical depth curves for the longest-lived MC case $\left(\tau_{\mathrm{DSNMS}}=6 \mathrm{Myr}\right)$ in Figure 6. In this case, the smaller variations in reionization history have a correspondingly smaller (and opposite) effect on $\tau_{\mathrm{e}}$, leading to slightly larger optical depths than in the $f_{\mathrm{DS}}=0$ case.

We also show in Figures 4 and 6 the $1 \sigma$ measurement of the integrated optical depth to last scattering from WMAP7 $\left(\tau_{\mathrm{e}}=0.088 \pm 0.014\right.$; Komatsu et al. 2011), along with a projected Planck sensitivity to the same quantity (Colombo et al. 

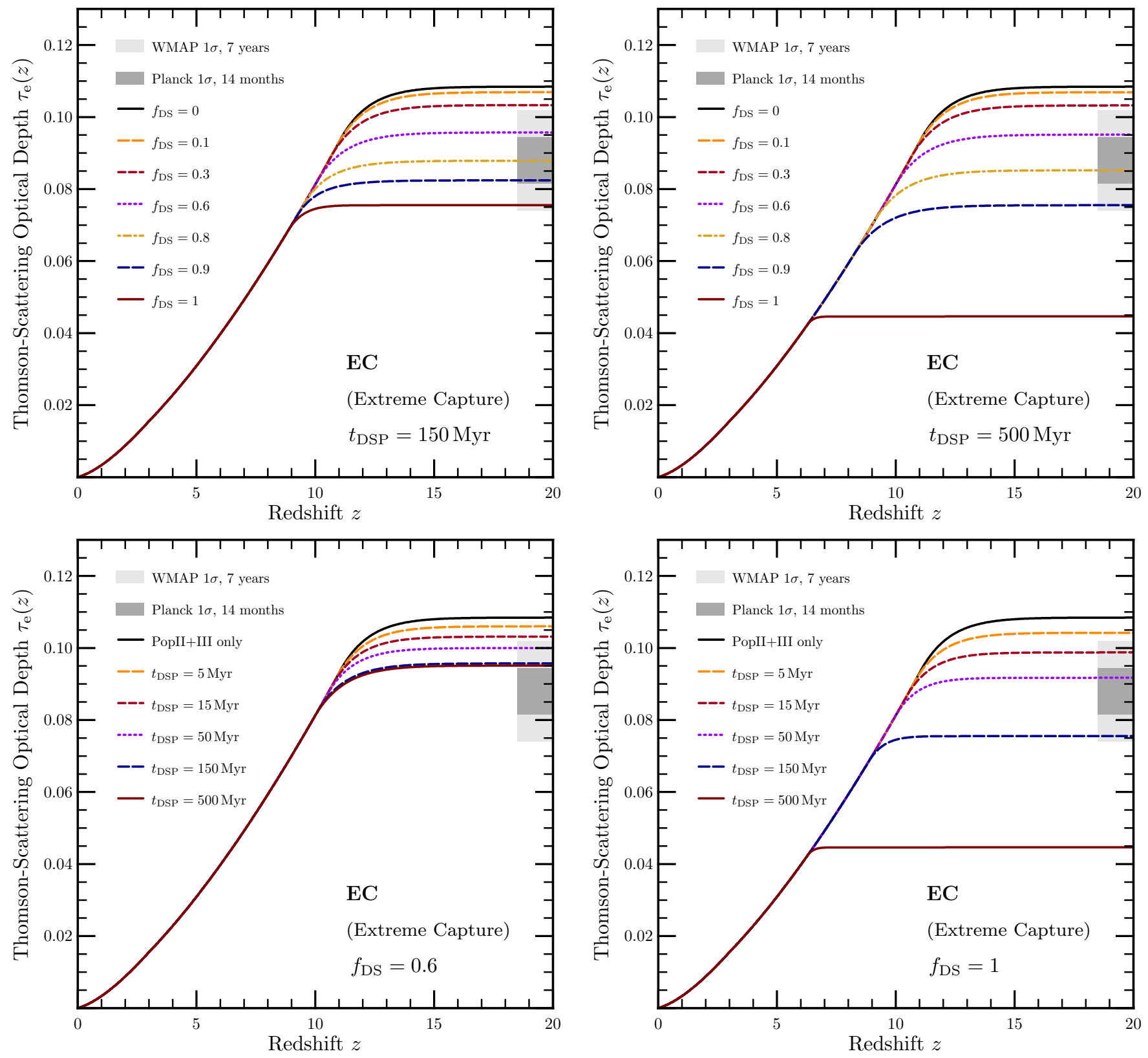

Figure 4. Evolution of the lookback optical depth from the present day to redshift $z$ according to Equation (6), for EC dark stars of varying lifetimes and abundances. These curves, and the corresponding dark stellar populations, correspond to the reionization histories presented in Figure 1. Larger dark star fractions and more extended lifetimes reionize later, producing smaller optical depths. For comparison, we show the WMAP7 measured $1 \sigma$ band (Komatsu et al. 2011) for the optical depth to the surface of last scattering, and the corresponding error band expected from Planck (Colombo et al. 2009), assuming it measures the same central value. While these strictly correspond to a redshift $z \sim 1090$, the optical depth curves have largely begun to plateau by $z \sim 20$ anyway.

(A color version of this figure is available in the online journal.)

2009), assuming the two experiments measure the same central value. ${ }^{9}$ Assuming that our chosen astrophysical reionization parameters in our canonical models are correct $\left(f_{\star} f_{\text {esc }}=0.005\right)$, a significant part of the more extreme end of the parameter space is already ruled out at better than a standard deviation by the WMAP7 measurement of $\tau_{\mathrm{e}}$; much more will be excluded by Planck. This is summarized in Figure 7, where we plot the $1 \sigma$ exclusion curves implied by WMAP7 and Planck measurements of $\tau_{\mathrm{e}}$ in the $t_{\mathrm{DSP}}-f_{\mathrm{DS}}$ plane, for the EC scenario.

\footnotetext{
9 The result we use from Colombo et al. (2009) is in fact the ratio of $1 \sigma$ uncertainties on the measured value of $\tau_{\mathrm{e}}$ obtainable with WMAP5 and Planck in the absence of foregrounds. We thus also assume the same percentage degradation in accuracy for both WMAP5 and Planck when mapping from expected results without foregrounds to final limits.
}

Noticeably, however, even the standard $f_{\mathrm{DS}}=0$, Pop III+II model exhibits tension with the WMAP7 data at more than the $1 \sigma$ level. As we discuss in Section 6.3, this should be taken with something of a grain of salt: similar variations in optical depth can be produced by reasonable changes in astrophysical parameters, so this should not be considered evidence for the existence of dark stars at this stage, even at the $1 \sigma$ level. Robust constraints could be obtained by full joint parameter scans of cosmological and (dark) reionization models. While such an exercise is well beyond the scope of this paper, the results we present in this section clearly indicate that dark stars can have a significant impact on the reionization history of the universe, and therefore the integrated optical depth of the CMB. 


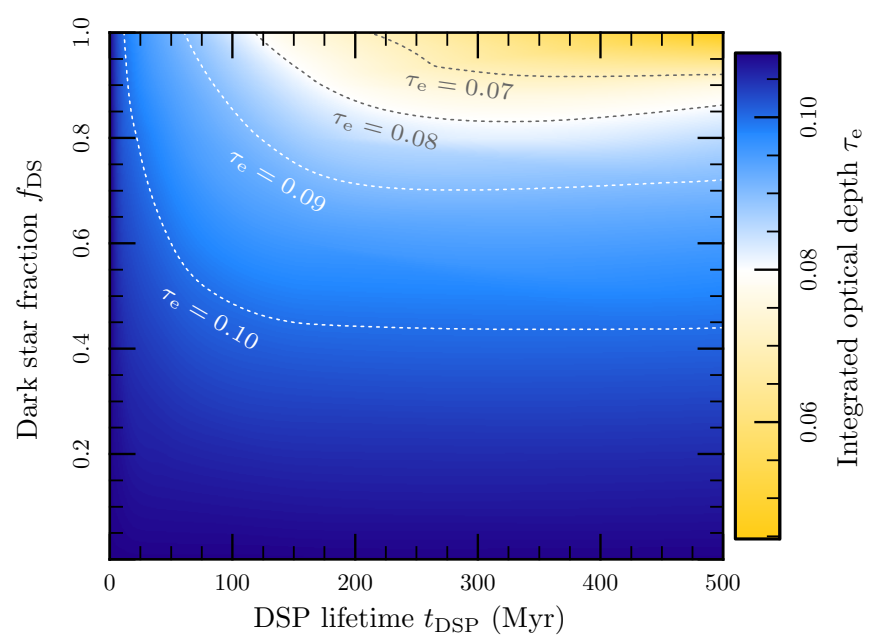

Figure 5. Contours of equal integrated CMB optical depth to $z=1090$ in the EC scenario, as a function of $f_{\mathrm{DS}}$ and $t_{\mathrm{DSP}}$. Here we performed the interpolation on the optical depths in Table 1 using two-dimensional exponential tension splines (Renka 1996b), based on a Delauney triangulation (Renka 1996a) and an iterative determination of the appropriate tension factors. Longer lifetimes and larger dark star fractions generically lead to smaller integrated optical depths; the slight upturn at large $t_{\mathrm{DSP}}$ in the $\tau_{\mathrm{e}}=0.08$ and 0.09 contours is an artifact of the interpolation.

(A color version of this figure is available in the online journal.)

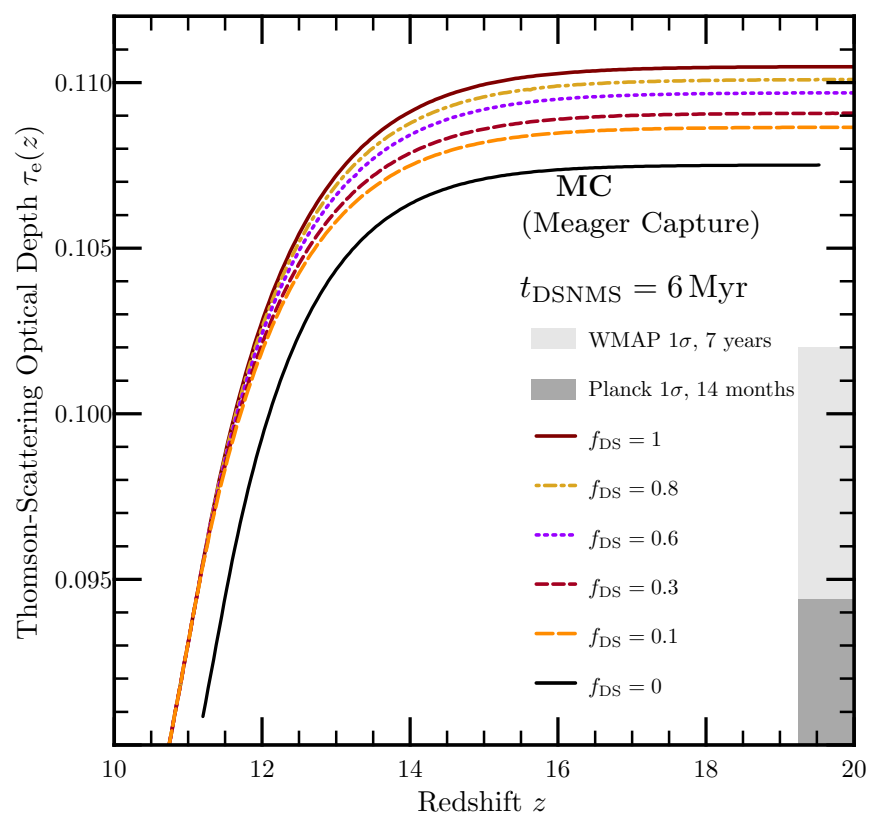

Figure 6. Evolution of the lookback optical depth from the present day to redshift $z$ according to Equation (6), for MC dark stars with the maximum allowed DSNMS lifetime ( $t_{\mathrm{DSNMS}}=6 \mathrm{Myr}$ ). These curves, and the corresponding dark stellar populations, correspond to the reionization histories presented in the right panel of Figure 2. In the MC scenario, earlier reionization caused by increasing values of $f_{\mathrm{DS}}$ results in a slight increase in optical depth. We also show WMAP7 and Planck $1 \sigma$ detection/prediction bands, as per Figure 4. Note the zoomed-in axes relative to Figure 4.

(A color version of this figure is available in the online journal.)

\subsection{Polarization}

We calculate the effects of the different reionization histories on the polarization (EE) CMB power spectra by modifying the Boltzmann code $\mathrm{CAMB}^{10}$ (Lewis et al. 2000). Instead of

\footnotetext{
10 http://camb.info
}

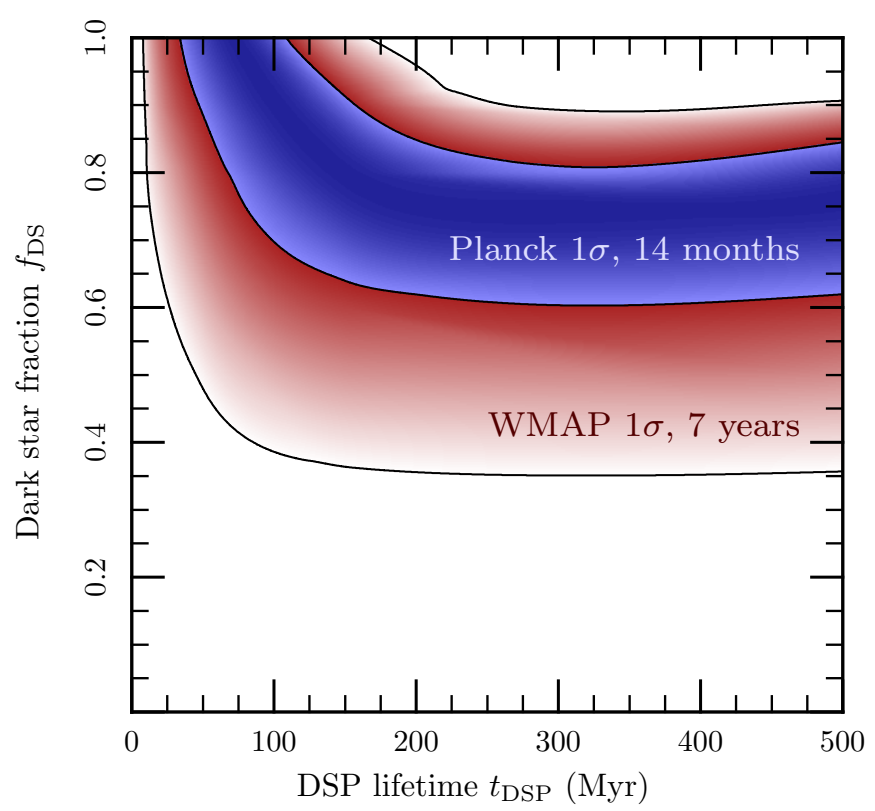

Figure 7. Implied $1 \sigma$ detection/exclusion regions in the $f_{\mathrm{DS}}-t_{\mathrm{DSP}}$ plane for EC dark stars, based on the integrated optical depth to last scattering observed by WMAP7 (Komatsu et al. 2011). We also show projected Planck constraints (Colombo et al. 2009), assuming that the same central value $\left(\tau_{\mathrm{e}}=0.088\right)$ is measured by Planck as by WMAP7. To guide the eye, the depth of shading is proportional to the likelihood of each parameter combination. For a product $f_{\star} f_{\text {esc }}=0.005$ of the star formation efficiency and UV photon escape fraction, parameter combinations outside the red WMAP shaded region are excluded at greater than $1 \sigma$ by existing data. Combinations outside the shaded blue region will be excludable at better than $1 \sigma$ by Planck. Note, however, that variations in $f_{\star} f_{\text {esc }}$ will shift these regions substantially; refer to discussions in the final paragraph of Section 6.1 and in Section 6.3. The same interpolation methods were employed in this figure as in Figure 5; the slight upturn of the boundaries of the Planck region at large $t_{\mathrm{DSP}}$ is again an artifact of the interpolation.

(A color version of this figure is available in the online journal.)

the simple hydrogen reionization model included in CAMB, our modified version uses the $\mathrm{H}$ ionization fractions presented in Section 5 as the basis for its reionization calculations. We include contributions to the total electron fraction from $\mathrm{H}$ II, He II, and He III with the assumptions stated earlier, as well as a residual electron fraction. As in the standard CAMB reionization calculation, we assume that electrons from He II track those from $\mathrm{H}$ II, and model contributions from He III with a smoothed step function centered at $z \sim 3.5$. We take the residual electron fraction after recombination to be $x_{\mathrm{e}}=2.1 \times 10^{-4}$, based on the output of RECFAST within CAMB. Using CAMB's highest accuracy setting, we calculated the temperature (TT), polarization (EE), and cross (TE) power spectra, as well as integrated optical depths. We checked that the optical depths computed with CAMB agree to within their stated numerical accuracy with those from Equation (6) (as presented in Table 1), and verified that the slight difference in the treatment of He III here and in Section 6.1 has a negligible impact on integrated optical depths.

In Figure 8, we plot EE polarization spectra for the same EC cases as illustrated in Figures 1 and 4. The spectra are normalized to the corresponding EE spectrum obtained in the standard $f_{\mathrm{DS}}=0$, Pop III+II scenario, in order to investigate the ability of CMB experiments to distinguish dark stars from standard reionization. To this end, we also plot the uncertainty on the normalization due to cosmic variance, and the combination of cosmic variance and total readout noise expected across the 70,100 , and $143 \mathrm{GHz}$ channels in the first 14 months of 

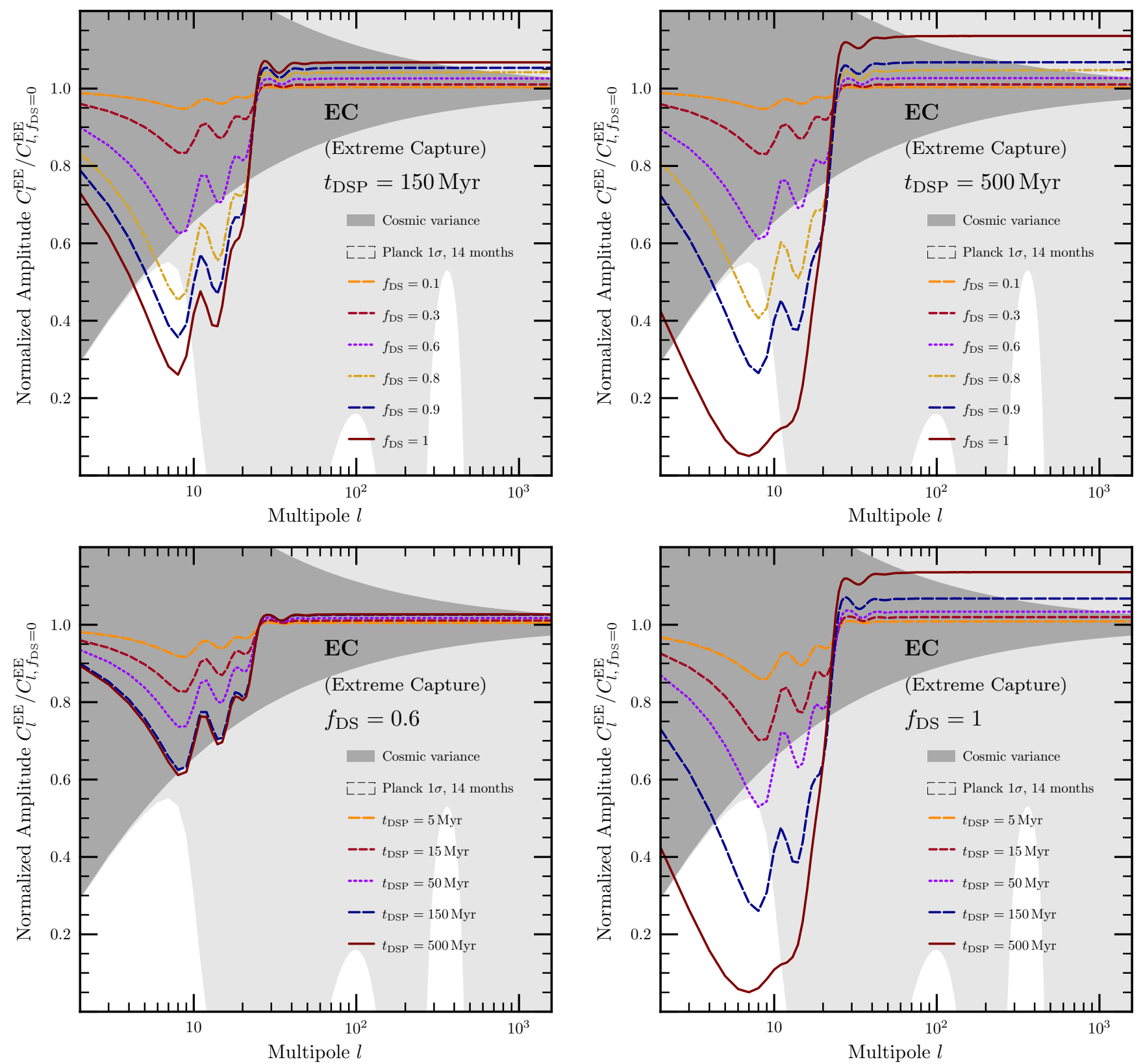

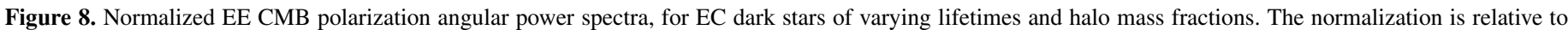

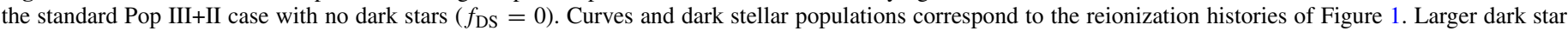

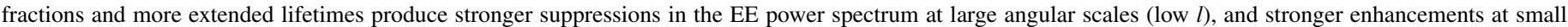

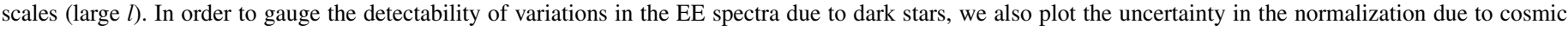

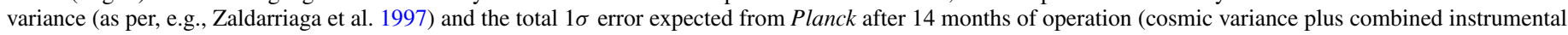
noise in 70, 100, and $143 \mathrm{GHz}$ channels; Colombo et al. 2009).

(A color version of this figure is available in the online journal.)

Planck operation (Colombo et al. 2009). Large parts of the parameter space are distinguishable from $f_{\mathrm{DS}}=0$ in a cosmicvariance-limited experiment, and a number of the more extreme scenarios are even detectable by Planck at better than $1 \sigma$. Although essentially all such models may be disfavored anyway by Planck's measurement of the integrated optical depth, the EE spectrum would nonetheless provide a complementary (albeit weak) statistical handle via which to increase the power of full parameter scans to exclude such dark star models.

We do not show TT or TE spectra for the EC scenario, as they exhibit less striking deviations from the corresponding spectra of the standard Pop III+II scenario at low multipoles $l$ (large angular scales) than the EE curves do. We do point out, however, that the TT and TE spectra exhibit damping at large $l$ due to the changing optical depth, which is more clearly visible than in the EE spectra. We also do not show power spectra for the MC or NC cases, as they show little deviation in general from the standard Pop III+II case.

\subsection{Astrophysical Uncertainties and Implications for Parameters of Reionization Models}

In Figure 9, we show the impacts of varying astrophysical parameters on CMB observables. Here we give the variations in optical depth and EE polarization resulting from the same variations of $f_{\star} f_{\text {esc }}$ as in Figure 3. For EE spectra, we normalize 

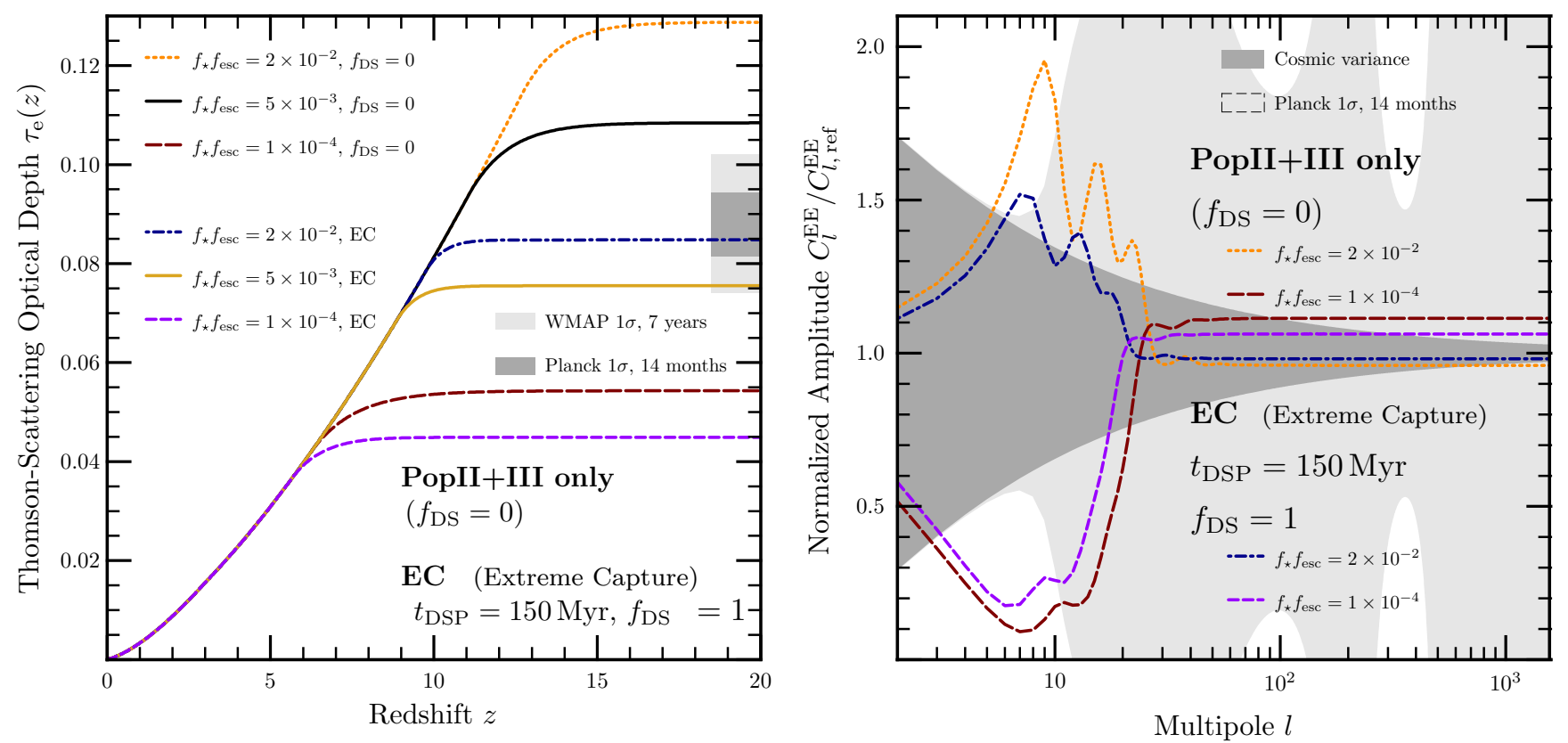

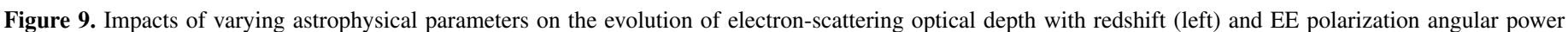

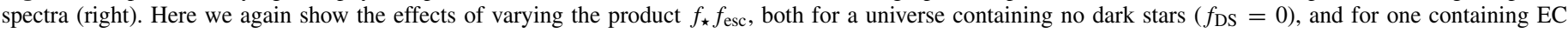

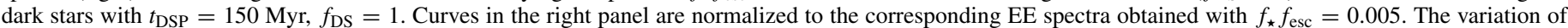

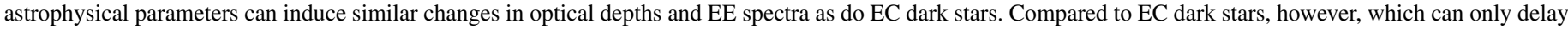
reionization, changes in $f_{\star}$ and $f_{\text {esc }}$ can also speed up reionization, leading to larger optical depths and enhanced large-scale (low $l$ ) power in the EE spectrum.

(A color version of this figure is available in the online journal.)

to the corresponding curve with default astrophysical parameters in each case; i.e., to the standard $f_{\star} f_{\text {esc }}=0.005$ Pop III+II spectrum for the $f_{\mathrm{DS}}=0$ curves, and to the $f_{\mathrm{DS}}=1$, $t_{\mathrm{DSP}}=150 \mathrm{Myr}, f_{\star} f_{\mathrm{esc}}=0.005$ spectrum for the curves where $f_{\mathrm{DS}}=1, t_{\mathrm{DSP}}=150 \mathrm{Myr}$. Comparing with Figures 4 and 8 , we see again that variations in the astrophysical parameters within reasonable ranges can have similar effects (both in strength and character) to variations in the dark star parameters. Although this means that the impact of dark stars on the CMB is very difficult to unambiguously disentangle from existing theoretical uncertainties in reionization modeling, it also serves as a clear indication that the potential effect of dark stars on CMB observables affected by reionization could be quite significant.

In some cases, it is possible that specific regions of the reionization parameter space that are ruled out in standard Pop III+II-only scenarios by the current WMAP7 (or projected Planck) data, are reopened by the possibility of having dark stars. For example, Figure 9 reveals that the extreme cases of varying astrophysical parameters $\left(f_{\star} f_{\text {esc }}=0.02\right.$ or $\left.10^{-4}\right)$ are ruled out for the Pop III+II-only scenarios by current CMB data. However, the case of $f_{\star} f_{\text {esc }}=0.02$ is not ruled out for the EC dark star case with $f_{\mathrm{DS}}=1, t_{\mathrm{DSP}}=150 \mathrm{Myr}$. Having more dark stars in the EC scenarios mimics a reduction in the astrophysical efficiency-i.e., increasing $f_{\mathrm{DS}}$ allows for greater astrophysical efficiency (larger $f_{\star}$, larger $f_{\text {esc }}$ or both) in reionization models. This is potentially an important factor to consider when placing constraints on the astrophysical aspects of reionization from CMB data (Haiman \& Holder 2003; Shull \& Venkatesan 2008).

The impacts of uncertainties in cosmological parameters on $\tau_{\mathrm{e}}$ are subdominant to those from astrophysical uncertainties. For the same two scenarios shown in Figure 9 (no dark stars and EC dark stars with $f_{\mathrm{DS}}=1$ and $t_{\mathrm{DSP}}=150 \mathrm{Myr}$ ), we find that varying $\sigma_{8}$ over the $1 \sigma$ allowed range of the WMAP7 fit we used as input for all calculations $\left(\sigma_{8}=0.801 \pm 0.030\right)$, leads to a change in the redshift of reionization of less than 1 , and a change of no more than 0.01 in $\tau_{e}$. This is relatively small compared with the variations in Figure 9.

\section{CONCLUSIONS}

We have calculated reionization histories, CMB optical depths, and anisotropy power spectra for a broad range of stellar population models containing dark stars. We identified three distinct regimes: no capture (NC), where dark stars live as cool, semi-diffuse objects for no more than $\sim 0.4 \mathrm{Myr}$ and then quickly move on to the main sequence; meager capture (MC), where dark stars undergo the same initial evolution as in the NC case, but exhibit slightly decreased surface temperatures and moderately increased lifetimes on the main sequence; and extreme capture (EC), where dark stars live an extended life as cool, semi-diffuse objects before reaching the main sequence.

$\mathrm{NC}$ dark stars have effectively no impact on reionization or its signatures in the CMB. MC dark stars cause the universe to reionize more quickly the longer lived and more numerous they are, producing a slight increase in the redshift of reionization. This leads to small increases in the total integrated optical depth of the CMB.

EC dark stars can have dramatic and completely opposite effects to MC dark stars, delaying reionization to as late as $z \sim 6$. Greater numbers of EC dark stars and longer dark star lifetimes lead to increased delays, and correspondingly decreased integrated optical depths to the last scattering surface of the CMB. Using WMAP7 observations of the integrated optical depth, we have been able to rule out more extreme areas of the parameter space covered by the EC scenario. Planck will improve these bounds significantly. EC dark stars also produce a characteristic suppression of EE polarization power on the 
largest scales of the $\mathrm{CMB}$, as well as a slight enhancement at small scales due to the decreased optical depth.

Many of these effects can be mimicked or compensated for by changes in the astrophysical parameters of standard reionization models. Disentangling the impact of dark stars from other theoretical uncertainties in reionization modeling will be challenging, even with Planck. Not only can dark stars have a substantial impact on reionization and its signatures in the CMB, but the addition of dark stars to standard reionization models can in fact substantially increase the range of astrophysical parameters that can be made consistent with existing (and future) observations.

P.S. thanks the organizers of the Cosmic Radiation Fields 2010 Workshop at DESY Hamburg, for providing a stimulating environment for discussion of an early version of these results, Dominik Schleicher and Erik Zackrisson in particular for such discussions, and the Department of Physics and Astronomy at the University of San Francisco, the Department of Physics at Stockholm University and the Max Planck Institute for Astrophysics for their hospitality while this work was being completed. P.S. is supported by the Lorne Trottier Chair in Astrophysics and an Institute for Particle Physics Theory Fellowship. A.V. gratefully acknowledges support from Research Corporation through the Single Investigator Cottrell College Science Award, and from the University of San Francisco Faculty Development Fund. The work of P.G. was supported in part by NSF award PHY-0456825 and NASA contract NNX09AT70G. E.P. thanks Loris Colombo for useful discussions, and acknowledges support from NASA grant NNX07AH59G and JPL Planck subcontract 1290790. G.H. acknowledges support from a Canada Research Chair, NSERC, and the Canadian Institute for Advanced Research.

\section{REFERENCES}

Abel, T., Bryan, G. L., \& Norman, M. L. 2002, Science, 295, 93

Benson, A. J., Sugiyama, N., Nusser, A., \& Lacey, C. G. 2006, MNRAS, 369, 1055

Bergström, L. 2000, Rep. Prog. Phys., 63, 793

Bertone, G. (ed.) 2010, Particle Dark Matter: Observations, Models and Searches (Cambridge: Cambridge Univ. Press)

Bertone, G., Hooper, D., \& Silk, J. 2005, Phys. Rep., 405, 279

Bouwens, R. J., Illingworth, G. D., Oesch, P. A., et al. 2010, ApJ, 708, L69

Bouwens, R. J., Illingworth, G. D., Oesch, P. A., et al. 2011, ApJ, submitted (arXiv:1105.2038)

Bromm, V., Kudritzki, R. P., \& Loeb, A. 2001, ApJ, 552, 464

Bromm, V., Yoshida, N., \& Hernquist, L. 2003, ApJ, 596, L135

Carilli, C. L. 2008, in Proc. Science, From Planets to Dark Energy: The Modern Radio Universe (PoS(MRU)014; Trieste, Italy: SISSA), 14

Casanellas, J., \& Lopes, I. 2009, ApJ, 705, 135

Chen, X., \& Miralda-Escudé, J. 2008, ApJ, 684, 18

Ciardi, B., Ferrara, A., Governato, F., \& Jenkins, A. 2000, MNRAS, 314, 611

Clark, P. C., Glover, S. C. O., Klessen, R. S., \& Bromm, V. 2011, ApJ, 727, 110

Colombo, L. P. L., Pierpaoli, E., \& Pritchard, J. R. 2009, MNRAS, 398, 1621

Dawson, S., Rhoads, J. E., Malhotra, S., et al. 2007, ApJ, 671, 1227

Fairbairn, M., Scott, P., \& Edsjö, J. 2008, Phys. Rev. D, 77, 047301

Fan, X., Strauss, M. A., Becker, R. H., et al. 2006, AJ, 132, 117

Freese, K., Bodenheimer, P., Spolyar, D., \& Gondolo, P. 2008a, ApJ, 685, L101

Freese, K., Gondolo, P., Sellwood, J. A., \& Spolyar, D. 2009, ApJ, 693, 1563

Freese, K., Ilie, C., Spolyar, D., Valluri, M., \& Bodenheimer, P. 2010, ApJ, 716, 1397

Freese, K., Spolyar, D., \& Aguirre, A. 2008b, J. Cosmol. Astropart. Phys., JCAP11(2008)014
Furlanetto, S. R., Oh, S. P., \& Briggs, F. H. 2006, Phys. Rep., 433, 181

Gnedin, N. Y. 2000, ApJ, 535, 530

Gould, A. 1987, ApJ, 321, 571

Haiman, Z., \& Holder, G. P. 2003, ApJ, 595, 1

Harker, G., Zaroubi, S., Bernardi, G., et al. 2010, MNRAS, 405, 2492

Heger, A., \& Woosley, S. E. 2002, ApJ, 567, 532

Hu, W., \& Dodelson, S. 2002, ARA\&A, 40, 171

Hubeny, I., \& Lanz, T. 1995, ApJ, 439, 875

Iocco, F. 2008, ApJ, 677, L1

Iocco, F., Bressan, A., Ripamonti, E., et al. 2008, MNRAS, 390, 1655

Jungman, G., Kamionkowski, M., \& Griest, K. 1996, Phys. Rep., 267, 195

Komatsu, E., Smith, K. M., Dunkley, J., et al. 2011, ApJS, 192, 18

Krauss, L. M., Freese, K., Spergel, D. N., \& Press, W. H. 1985, ApJ, 299, 1001

Krumholz, M. R., Klein, R. I., McKee, C. F., Offner, S. S. R., \& Cunningham, A. J. 2009, Science, 323, 754

Larson, D., Dunkley, J., Hinshaw, G., et al. 2011, ApJS, 192, 16

Leitherer, C., Ferguson, H. C., Heckman, T. M., \& Lowenthal, J. D. 1995, ApJ, 454, L19

Lewis, A., Challinor, A., \& Lasenby, A. 2000, ApJ, 538, 473

Loeb, A. 2009, in Astrophysics in the Next Decade, ed. H. A. Thronson, M. Stiavelli, \& A. Tielens (Ap\&SS Proc.; Netherlands: Springer), 481

Mapelli, M., Ferrara, A., \& Pierpaoli, E. 2006, MNRAS, 369, 1719

McKee, C. F., \& Tan, J. C. 2008, ApJ, 681, 771

Mitchell, D., Greenhill, L. J., Clark, M., et al. 2010, in RFI Mitigation Workshop, Proceedings of Science (PoS(RFI2010)016; Trieste, Italy: SISSA), 16

Morales, M. F., \& Wyithe, J. S. B. 2010, ARA\&A, 48, 127

Natarajan, A., Tan, J. C., \& O'Shea, B. W. 2009, ApJ, 692, 574

Ohkubo, T., Nomoto, K., Umeda, H., Yoshida, N., \& Tsuruta, S. 2009, ApJ, 706, 1184

Peters, T., Klessen, R. S., Mac Low, M.-M., \& Banerjee, R. 2010, ApJ, 725, 134

Press, W. H., \& Spergel, D. N. 1985, ApJ, 296, 679

Renka, R. J. 1996a, ACM Trans. Math. Softw., 22, 1

Renka, R. J. 1996b, ACM Trans. Math. Softw., 22, 9

Ripamonti, E., Iocco, F., Ferrara, A., et al. 2010, MNRAS, 406, 2605

Ripamonti, E., Mapelli, M., \& Ferrara, A. 2007, MNRAS, 375, 1399

Salati, P., \& Silk, J. 1989, ApJ, 338, 24

Samtleben, D., Staggs, S., \& Winstein, B. 2007, Ann. Rev. Nucl. Part. Sci., 57, 245

Schaerer, D. 2002, A\&A, 382, 28

Schleicher, D. R. G., Banerjee, R., \& Klessen, R. S. 2009, Phys. Rev. D, 79, 043510

Schleicher, D. R. G., Banerjee, R., Sur, S., et al. 2010, A\&A, 522, A115

Scott, P. 2011, in Cosmic Radiation Fields 2010, Proceedings of Science (PoS(CRF 2010)021; Trieste, Italy: SISSA), 21

Scott, P., Edsjö, J., \& Fairbairn, M. 2008, in Proceedings of Dark Matter in Astroparticle and Particle Physics: Dark 2007, ed. H. K. KlapdorKleingrothaus \& G. F. Lewis (Singapore: World Scientific), 387

Scott, P., Fairbairn, M., \& Edsjö, J. 2009, MNRAS, 394, 82

Shull, J. M., \& Venkatesan, A. 2008, ApJ, 685, 1

Sivertsson, S., \& Gondolo, P. 2011, ApJ, 729, 51

Spolyar, D., Bodenheimer, P., Freese, K., \& Gondolo, P. 2009, ApJ, 705, 1031

Spolyar, D., Freese, K., \& Gondolo, P. 2008, Phys. Rev. Lett., 100, 051101

Stacy, A., Greif, T. H., \& Bromm, V. 2010, MNRAS, 403, 45

Stasielak, J., Biermann, P. L., \& Kusenko, A. 2007, ApJ, 654, 290

Steigman, G., Quintana, H., Sarazin, C. L., \& Faulkner, J. 1978, AJ, 83, 1050

Sur, S., Schleicher, D. R. G., Banerjee, R., Federrath, C., \& Klessen, R. S. 2010, ApJ, 721, L134

Tumlinson, J., Shull, J. M., \& Venkatesan, A. 2003, ApJ, 584, 608

Tumlinson, J., Venkatesan, A., \& Shull, J. M. 2004, ApJ, 612, 602

Turk, M. J., Abel, T., \& O’Shea, B. 2009, Science, 325, 601

Umeda, H., Yoshida, N., Nomoto, K., et al. 2009, J. Cosmol. Astropart. Phys., JCAP08(2009)024

Venkatesan, A., \& Truran, J. W. 2003, ApJ, 594, L1

Venkatesan, A., Tumlinson, J., \& Shull, J. M. 2003, ApJ, 584, 621

Wada, K., \& Venkatesan, A. 2003, ApJ, 591, 38

Wise, J. H., \& Abel, T. 2008, ApJ, 684, 1

Wyithe, J. S. B., \& Loeb, A. 2003, ApJ, 586, 693

Yoon, S.-C., Iocco, F., \& Akiyama, S. 2008, ApJ, 688, L1

Zackrisson, E., Scott, P., Rydberg, C.-E., et al. 2010a, ApJ, 717, 257

Zackrisson, E., Scott, P., Rydberg, C.-E., et al. 2010b, MNRAS, 407, L74

Zaldarriaga, M., Spergel, D. N., \& Seljak, U. 1997, ApJ, 488, 1 